Universidade de São Paulo

Instituto de Física

\title{
A nuvem mesônica e os fatores de forma estranhos do próton
}

\author{
Daniela Morales Tolentino Leite \\ Orientadora: Profa. Dra. Marina Nielsen \\ Dissertação apresentada ao Instituto de \\ Física da Universidade de São Paulo \\ para a obtenção do título de Mestre em \\ Ciências
}

Banca Examinadora:

Profa. Dra. Marina Nielsen (IFUSP) - Orientadora

Prof. Dr. Manoel Roberto Robilotta (IFUSP)

Prof. Dr. Tobias Frederico (ITA)

São Paulo, 2008 
FICHA CATALOGRÁFICA

Preparada pelo Serviço de Biblioteca e Informação do Instituto de Física da Universidade de São Paulo

Leite, Daniela Morales Tolentino

A nuvem mesônica e os fatores de forma estranhos do próton - São Paulo - 2008

Dissertação (Mestrado) - Universidade de São Paulo. Instituto de Física - Depto. de Física Experimental

Orientador: Profa. Dra. Marina Nielsen

Área de Concentração: Física de Hádrons

Unitermos: 1. Física de Partículas; 2. Física de Hádrons; 3. Métodos não-perturbativos; 4. Estranheza do Nucleon; 5. Modelo da nuvem mesônica. 


\section{Agradecimentos}

- À Prof. Marina, por incentivar o meu interesse em física de hádrons desde o grupo de iniciação científica e por ter me orientado no mestrado.

- Aos alunos e professores do GRHAFITE, pela amizade e por tornarem nosso ambiente de trabalho um lugar agradável e descontraído. Agradeço especialmente ao Prof. Fernando, pela ajuda com o mestrado e com outras dúvidas relacionadas à minha carreira acadêmica, e também ao Gabriel, ao David, ao Ricardo e à Patrícia, por me ajudarem a esclarecer muitas das questões que foram surgindo nestes dois anos.

- Ao Marcello e ao Marcus, pela grande amizade que temos e por aguentarem minhas reclamações desde o começo da graduação.

- Ao Mark, pelos momentos felizes que me propiciou nestes últimos meses.

- Aos meus irmãos, Pedro e Gabriela, por apoiarem todos os meus projetos e compartilharem comigo a alegria das conquistas.

- Às pessoas que mais amo, Pedro e Iracy, que com muita dedicação me ensinaram o que eu de fato precisava saber para poder aprender o restante.

- E por fim, agradeço à FAPESP pelo apoio financeiro, sem o qual este trabalho não poderia ter sido realizado. 


\section{Resumo}

O objetivo deste trabalho foi incluir o méson escalar $\kappa$ na núvem de mésons estranhos do próton e verificar se, desta forma, a contribuição de estranheza para as suas propriedades eletromagnéticas poderia ser explicada pelo modelo da núvem mesônica. Os observáveis que quantificam tal contribuição são os fatores de forma estranhos elétrico $\left(G_{E}^{s}\right)$ e magnético $\left(G_{M}^{s}\right)$, que têm sido objeto de grande interesse experimental nos últimos 10 anos.

Usando a versão da nuvem que inclui o méson $\kappa$, nós calculamos $G_{E}^{s}$ e $G_{M}^{s}$ em função do momento transferido dentro do intervalo $0 \leq Q^{2} \leq 1,2 \mathrm{GeV}^{2}$, de modo a abranger toda a gama de dados disponíveis no momento. Comparamos nossos resultados com os dados existentes para $G_{E}^{s}$ e $G_{M}^{s}$ e encontramos um ótimo acordo entre experimento e modelo, demonstrando que a inclusão do $\kappa$ na núvem de mésons do próton é fundamental para que o seu conteúdo de estranheza possa ser compreendido. 


\section{Abstract}

The goal of this work was to include the scalar $\kappa$ meson on the meson cloud of the proton, and then to verify if the strangeness contribution to the electromagnetic properties of the proton could be explained by the meson cloud model. The observables that quantify such a contribution are the electric $\left(G_{E}^{s}\right)$ and magnetic $\left(G_{M}^{s}\right)$ strange form factors, which have been subject of great experimental interest in the last 10 years.

Using the version of the cloud which includes the $\kappa$ meson, we calculated $G_{E}^{s}$ and $G_{M}^{s}$ as a function of the transferred momentum in the interval $0 \leq Q^{2} \leq 1.2 \mathrm{GeV}^{2}$, to cover the full range of available data at the time. We compared our results with existing data for $G_{E}^{s}$ and $G_{M}^{s}$ and we found a good agreement between experiment and model, showing that including $\kappa$ on the meson cloud of the proton is crucial to understand its strangeness content. 


\section{Sumário}

Agradecimentos $\quad$ i

Resumo

Abstract $\quad$ iii

1 Introdução 1

2 Fatores de forma estranhos $\quad 5$

2.1 Amplitude de espalhamento $e p \rightarrow e p \ldots \ldots \ldots$. . . . . . . . . 5

2.2 Fatores de forma do próton . . . . . . . . . . . . . 8

2.2.1 Interação Eletromagnética . . . . . . . . . . . . . . . . . . 8

2.2 .2 Interação Fraca . . . . . . . . . . . . . . . . . . . . . . . . 10

2.2.3 Fatores de forma e propriedades estáticas . . . . . . . . . . . . . 11

2.2.4 Decomposição dos fatores de forma nas contribuições dos quarks . . 12

2.3 Contribuição do quark estranho . . . . . . . . . . . . . . . . . . . . 15

2.4 Determinação experimental de $G_{E}^{s}$ e $G_{M}^{s} \ldots \ldots \ldots$. . . . . . . . . 16

2.4.1 $G_{E}^{s}$ e $G_{M}^{s}$ em função de observáveis . . . . . . . . . . . . . . 16

2.4.2 Experimentos de violação de paridade em espalhamento ep $\rightarrow e p$. . 17

3 Modelo da nuvem mesônica $\quad 21$

3.1 A nuvem de mésons estranhos . . . . . . . . . . . . . . . . . . 21

3.2 Calculando $G_{E}^{s}$ e $G_{M}^{s}$ no contexto da nuvem . . . . . . . . . . . . . . . 23

3.3 Acoplamento do fóton com o próton do MCM . . . . . . . . . . . . 25

3.3.1 Acoplamentos pontuais com os estados virtuais . . . . . . . . 25

3.3 .2 Função de vértice seagull . . . . . . . . . . . . . . . . . . 28

3.3 .3 Acoplamento com o vértice não pontual . . . . . . . . . . . . . 31

3.4 Incluindo a contribuição do $\Sigma \ldots \ldots . \ldots$. . . . . . . . . . 33

3.5 Amplitude total do processo . . . . . . . . . . . . . . . . 35 
4 Cálculo dos fatores de forma estranhos $\quad 37$

4.1 Acoplamento bariônico . . . . . . . . . . . . . . . . . . . . 37

4.2 Acoplamento mesônico . . . . . . . . . . . . . . . . . . . . . . . 41

4.3 Acoplamento com o vértice não pontual . . . . . . . . . . . . . . . . . . . 43

5 Resultados numéricos $\quad 46$

5.1 Constantes e parâmetros . . . . . . . . . . . . . . . 46

5.2 Fator de forma elétrico estranho . . . . . . . . . . . . . . . . . . . 48

5.3 Fator de forma magnético estranho . . . . . . . . . . . . . . . 50

5.4 Combinação $G_{E}^{s}+\eta G_{M}^{s} \ldots \ldots \ldots \ldots \ldots \ldots$

6 Conclusão $\quad 54$

A Demonstração da Eq. (3.18) 56

B Relações úteis $\quad 58$ 


\section{Lista de Figuras}

2.1 Diagramas de Feynman em primeira ordem para o espalhamento elástico $e p$. Os momentos iniciais (finais) do elétron e do próton são $k$ e $p$ ( $k^{\prime}$ e $\left.p^{\prime}\right)$, respectivamente. O momento carregado pelo bóson trocado é definido como $q^{\prime} \equiv p^{\prime}-p=k-k^{\prime} \ldots \ldots \ldots \ldots \ldots$. . . . . . . . . . 6

3.1 Representação pictórica do mecanismo do modelo da nuvem mesônica. . . 22

3.2 Diagrama de Feynman para o processo básico do modelo da nuvem mesônica. A linha tracejada representa um méson estranho $M(M=K, \kappa)$ e a linha contínua interna, um híperon $Y(Y=\Lambda, \Sigma)$. As linhas externas representam o próton. . . . . . . . . . . . . . 22

3.3 Diagrama de Feynman que representa o acoplamento do fóton com a componente estranha do próton. . . . . . . . . . . . . . . . . . . 23

3.4 Diagramas de Feynman para o acoplamento do fóton com o híperon (a) e com o méson (b) virtuais. As linhas contínuas interna e externa representam o híperon e o próton, respectivamente. A linha pontilhada representa o méson $\kappa \ldots \ldots \ldots \ldots \ldots$. . . . . . . . . . . . . . . .

3.5 Idem à Fig. 3.4, mas agora considerando o acoplamento do fóton com o vértice extenso $N \kappa \Lambda$. . . . . . . . . . . . . . . . . . . . 32

3.6 Diagramas de Feynman que contribuem para a amplitude total $\Gamma_{\mu}^{s}$ invariante de gauge. As linhas contínuas interna e externa representam um híperon $Y(Y=\Lambda, \Sigma)$ e um próton, respectivamente. A linha pontilhada representa o méson $\kappa \ldots \ldots \ldots \ldots \ldots \ldots$

5.1 Fator de forma elétrico estranho. As linhas azul e vermelha mostram nossos resultados com $\Lambda_{\kappa}=0,9 \mathrm{GeV}, \Lambda_{K}=1,1 \mathrm{GeV}$ e $\Lambda_{\kappa}=1,1 \mathrm{GeV}, \Lambda_{K}=0,9$ $\mathrm{GeV}$, respectivamente. . . . . . . . . . . . . . . 48

5.2 Contribuição do káon (linha tracejada) e do méson $\kappa$ (linha cheia) para o fator de forma elétrico estranho, calculada nas combinações de cut-offs I (acima) e II (abaixo) . . . . . . . . . . . . . . . . . . . 
5.3 Idem à Fig. 5.1, mas para o fator de forma magnético estranho. . . . . . . 50

5.4 Idem à Fig. 5.2, mas para o fator de forma magnético estranho. . . . . . . 51

5.5 Idem à Fig. 5.1, mas para a combinação $G_{E}^{s}+\eta G_{M}^{s}$ dos fatores de forma estranhos. . . . . . . . . . . . . . . . . . . . 52

5.6 Contribuição do káon (linha tracejada) e do méson $\kappa$ (linha cheia) para o fator de forma elétrico estranho, na escolha I de cut-offs. . . . . . . . . . . 52

5.7 Idem à Fig. 5.6, mas na escolha II de cut-offs. . . . . . . . . . . . . . . . 53 


\section{Lista de Tabelas}

2.1 As cargas elétromagnéticas e fracas dos férmions fundamentais do Modelo Padrão [25]. Nas expressões, $\theta_{W}$ denota o ângulo de Weinberg. . . . . . . . . 6 


\section{Capítulo 1}

\section{Introdução}

No primeiro modelo de quarks sugerido independentemente por Gell-mann [1] e Zweig [2], o próton era visto como um estado ligado de três férmions pontuais, com massas em torno de $300 \mathrm{MeV}$, que juntos podiam explicar todas as suas propriedades, tais como massa, carga e spin. No entanto, uma estrutura muito mais complexa tem sido evidenciada desde os primeiros experimentos de espalhamento inelástico profundo no SLAC-MIT [3]. Na descrição atual, em termos da Cromodinâmica Quântica (QCD), o próton é constituído pelos três quarks de valência uud, com massas próximas de $10 \mathrm{MeV}$, e também por glúons e por um mar de pares quark-antiquark $(\bar{q} q)$ que podem existir durante um tempo limitado pelo princípio de Heinsenberg. Este é o chamado mar de Dirac do próton.

Nesta descrição é possível que dentro do próton ocorra a formação de pares $\bar{q} q$ de qualquer sabor. Entretanto, a criação de pares estranhos é particularmente interessante, pois oferece uma possibilidade única de estudo dos efeitos do mar de Dirac nas propriedades do próton. Uma vez que este hádron não possui estranheza líquida, os efeitos dos pares $\bar{s} s$ provêm unicamente do mar, ao contrário do que acontece com os quarks $u$ e $d$. Além disso, ainda que a massa do quark estranho seja muito maior que a dos quarks de valência $\left(m_{s} \approx 100 \mathrm{MeV}\right)$ ela ainda é bem menor que a massa do próton $\left(m_{N} \approx 1 \mathrm{GeV}\right)$ e por isso a produção de estranheza pode ser substancial enquanto que as contribuições dos quarks $c, b$ e $t$ são desprezíveis. E isto é o que, de fato, vem sendo observado experimentalmente desde a década de 70 .

A primeira evidência de uma contribuição estranha não trivial veio da medida do termo $\sigma$ em espalhamento píon-nucleon [4], em 1976. É possível extrair a contribuição do quark $s$ para a massa do próton a partir do valor experimental de $\sigma_{\pi N}$ e, desde aquela 
época até hoje [5], o valor de $\sigma_{\pi N}(55 \sim 75 \mathrm{MeV})$ implica em uma contribuição estranha de pelo menos $130 \mathrm{MeV}$ para a massa total do próton.

Posteriormente, já no final da década de 80, os resultados da famosa experiência EMC [6] mostraram que os quarks de valência contribuem para menos que a metade do spin total do próton, e também sugeriram que uma parte significante do spin possa ser carregada por quarks estranhos. Experimentos subsequentes no CERN e no SLAC sustentam os resultados iniciais da EMC, e uma análise global dos dados [7] sugere que a contribuição de estranheza para o spin seja $\Delta s \approx-0,15$.

Face a estas evidências experimentais, surgiu a questão de qual seria então a influência da estranheza nas propriedades eletromagnéticas do próton, tais como distribuição de carga e momento magnético. Estas quantidades estão relacionadas com os fatores de forma eletromagnéticos, cuja contribuição estranha é dada pelo o que chamamos de fatores de forma eletromagnéticos estranhos, $G_{E}^{s}$ e $G_{M}^{s}$. Com base nesta discussão, Kaplan e Manohar publicaram em 1988 um trabalho sugerindo que seria possível determinar $G_{E}^{s}$ e $G_{M}^{s}$ fazendo a medida dos fatores de forma fracos vetoriais $G_{E}^{Z p}$ e $G_{M}^{Z p}$ [8]. Logo em seguida à publicação do artigo de Kaplan, McKeown e Beck escreveram sobre a possibilidade oferecida pelos experimentos de violação de paridade em espalhamento elástico ep que, quando combinados com as medidas existentes dos fatores de forma eletromagnéticos do próton, poderiam permitir a identificação dos fatores de forma fracos e, conseqüentemente, de $G_{E}^{s}$ e $G_{M}^{s}[9,10]$.

Todos estes trabalhos deram origem a um grande projeto experimental, que começou no final da década de 90 com as colaborações SAMPLE [11-13] no MIT e HAPPEX [1417] no TJNAF e que, posteriormente, contou com a Colaboração A4 [18, 19] no MAMI. Esses experimentos mediram os fatores de forma estranhos isoladamente e também dentro da combinação

$$
G_{E}^{s}\left(Q^{2}\right)+\eta\left(Q^{2}\right) G_{M}^{s}\left(Q^{2}\right)
$$

para alguns valores de momento trocado no intervalo de $0,09<Q^{2}<0,5 \mathrm{GeV}^{2}$. Na Eq. (1.1), $\eta\left(Q^{2}\right)=\tau G_{M}^{\gamma p} / \epsilon G_{E}^{\gamma p}, \operatorname{com} \epsilon=\left[1+2(1+\tau) \tan ^{2}(\theta / 2)\right]^{-1}$ e $\tau=Q^{2} / 4 m_{N}^{2}$, sendo que $G_{E, M}^{\gamma p}$ são os fatores de forma eletromagnéticos do próton e $\theta$ é o ângulo de espalhamento do elétron no referencial do laboratório. Em 2005, os resultados da Colaboração G0 no TJNAF [20] forneceram novos dados para a combinação em (1.1) sobre uma região de 
momentos transferidos relativamente extensa: $0,12 \leq Q^{2} \leq 1,0 \mathrm{GeV}^{2}$. Os dados de todos estes experimentos indicam uma dependência não trivial dos fatores de forma estranhos com $Q^{2}$ e apresentam um desafio para os modelos sobre a estrutura do próton.

Neste regime de baixas energias, espera-se que o mar do próton tenha uma forte componente estranha de origem não perturbativa, e uma possibilidade para explicar efeitos não perturbativos no mar é o modelo da nuvem mesônica (Meson Cloud Model - MCM). No modelo da nuvem, considera-se que um par quark-antiquark do mar de Dirac possa se acoplar com os quarks de valência, dando origem a estados formados por um méson e um bárion virtuais. Este mecanismo fornece uma explicação natural para que haja uma distribuição de carga estranha dentro do próton.

Em um dos trabalhos anteriores do grupo [21] foi usada uma versão do modelo da nuvem mesônica que incluía as contribuições do káon e do $K^{*}$ no cálculo dos fatores de forma estranhos sobre a região $0 \leq Q^{2} \leq 3,0 \mathrm{GeV}^{2}$. Comparando o resultado destes trabalhos com os dados experimentais concluiu-se que a única forma de se conseguir alguma concordância com os dados para a combinação em (1.1) é fazendo uma escolha de parâmetros que suprime fortemente a contribuição do $K^{*}$. Além disso, apesar de ser possível fixar os parâmetros do modelo de forma a obter uma consistência com os dados experimentais para a combinação, não é possível, com o mesmo conjunto de parâmetros, descrever os dados de $G_{E}^{s}$ e $G_{M}^{s}$ isoladamente.

Desta forma vemos que, quando se considera apenas os estados intermediários envolvendo um híperon e os mésons $K$ e $K^{*}$, o modelo da nuvem mesônica não é capaz de explicar todos os dados experimentais disponíveis no momento. Como sabemos que as contribuições provenientes dos híperons mais pesados não são importantes, nos resta investigar se a contribuição dos estados intermediários envolvendo o méson escalar $\kappa$ podem ser importantes. Este méson foi observado no decaimento não leptônico do méson $D$ através do forte aumento no canal de onda- $S$ do estado $K \pi$, com uma massa $m_{\kappa}=0,797 \pm 0,019 \pm 0,042 \mathrm{GeV}$ e uma largura $\Gamma_{\kappa}=0,410 \pm 0,043 \pm 0,085 \mathrm{GeV}[22]$.

Sendo assim, o objetivo deste trabalho foi incluir a contribuição do méson $\kappa$ na nuvem estranha do próton, e verificar se com isso os dados para os fatores de forma estranhos poderiam ser explicados pelo nosso modelo. Optamos por simplicidade começar com uma versão da nuvem que inclui somente o káon e o $\kappa$, visto que no trabalho anterior do grupo a concordância com os dados só era possível se a contribuição do $K^{*}$ fosse suprimida. Como 
os nossos resultados descreveram bem os dados experimentais, não houve a necessidade de incluir o káon vetorial no cálculo.

Existe também uma análise global, realizada por Pate et al. [23] na qual os dados das colaborações G0 e HAPPEX para $G_{E}^{s}+\eta G_{M}^{s}$ são analisados em conjunto com medidas da Colaboração E734 no BNL para o fator de forma estranho axial [24], fornecendo um conjunto de dados separados para os fatores de forma estranhos. Também comparamos os resultados desta análise com o nosso modelo. 


\section{Capítulo 2}

\section{Fatores de forma estranhos}

As propriedades eletromagnéticas e fracas do próton são observadas em espalhamento elástico ep e descritas por fatores de forma que parametrizam a amplitude deste processo. A contribuição da estranheza para tais propriedades é dada pelos chamados fatores de forma estranhos, $G_{E}^{s}$ e $G_{M}^{s}$. Neste capítulo, o nosso objetivo é discutir qual a definição destes observáveis, o tipo de experimento em que eles são medidos e a sua interpretação física.

\subsection{Amplitude de espalhamento $e p \rightarrow e p$}

A definição dos fatores de forma do próton aparece quando calculamos a amplitude de espalhamento $e p \rightarrow e p$. Tal processo, em primeira ordem na teoria de perturbação, pode acontecer de duas maneiras diferentes, representadas pelos diagramas de Feynman da Fig. 2.1: (a) por interação eletromagnética, com a troca de um único fóton $\gamma$, ou então (b) por interação fraca neutra, com a troca de um único bóson $Z$. Cada diagrama tem uma amplitude invariante associada, $\mathcal{M}_{\gamma}$ e $\mathcal{M}_{Z}$, e a soma destas duas resulta na amplitude total do processo.

Para calcular as amplitudes é preciso conhecer como as correntes do elétron e do próton se acoplam com os bósons de gauge das interações em questão. No caso do elétron pode-se partir diretamente do Modelo Padrão, que diz que os acoplamentos de férmions fundamentais com o $\gamma$ e o $Z$ são escritos como [25]

$$
\begin{gathered}
i e e_{f} \gamma^{\mu}, \\
i \frac{g M_{Z}}{4 M_{W}} \gamma^{\mu}\left(g_{V}^{f}+g_{A}^{f} \gamma^{5}\right),
\end{gathered}
$$




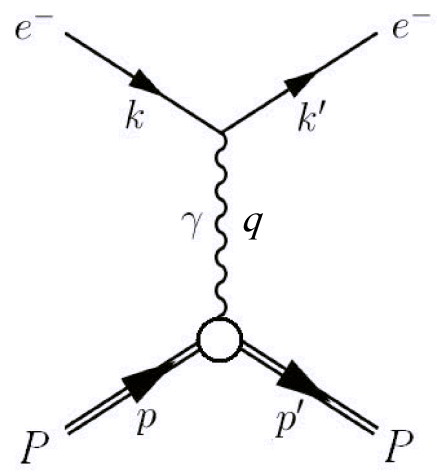

(a)

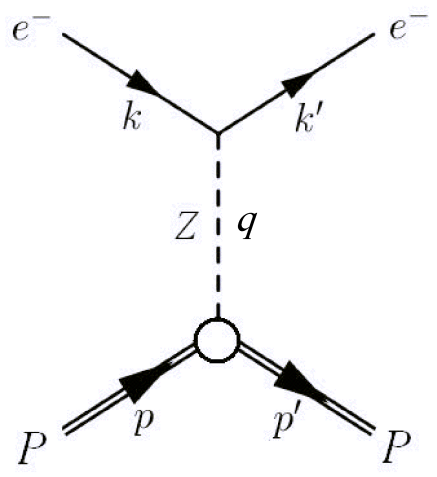

(b)

Figura 2.1: Diagramas de Feynman em primeira ordem para o espalhamento elástico ep. Os momentos iniciais (finais) do elétron e do próton são $k$ e $p\left(k^{\prime}\right.$ e $\left.p^{\prime}\right)$, respectivamente. O momento carregado pelo bóson trocado é definido como $q^{\prime} \equiv p^{\prime}-p=k-k^{\prime}$.

respectivamente. Nas expressões, e e $g$ são as constantes de acoplamento eletromagnético e fraco, $M_{Z}$ e $M_{W}$ são as massas dos bósons $Z$ e $W, g_{V}^{f}$ e $g_{A}^{f}$ são as cargas fracas vetorial e axial do férmion e $e_{f}$ a sua carga eletromagnética. As diversas cargas dos férmions fundamentais do Modelo Padrão estão na Tabela 2.1.

No caso do próton, não podemos mais usar as Eqs. (2.1) e (2.2), pois o próton possui estrutura e não é uma partícula fundamental de Dirac. Construímos os acoplamentos do próton com os bósons de gauge em analogia com o caso de férmions fundamentais, fazendo as substituições $e_{f} \gamma_{\mu} \rightarrow \Gamma_{\mu}^{\gamma}$ na Eq. (2.1) e $\gamma_{\mu}\left(g_{V}^{f}+g_{A}^{f} \gamma_{5}\right) \rightarrow\left(\Gamma_{\mu}^{V}+\Gamma_{\mu}^{A}\right)$ na Eq. (2.2), de modo a obter

Tabela 2.1: As cargas elétromagnéticas e fracas dos férmions fundamentais do Modelo Padrão [25]. Nas expressões, $\theta_{W}$ denota o ângulo de Weinberg.

\begin{tabular}{cccc}
\hline \hline Férmions & $e_{f}$ & $g_{V}^{f}$ & $g_{A}^{f}$ \\
\hline \hline$\nu_{e}, \nu_{\mu}, \nu_{\tau}$ & 0 & 1 & -1 \\
$e^{-}, \mu^{-}, \tau^{-}$ & -1 & $-1+4 \sin ^{2} \theta_{W}$ & 1 \\
$u, c, t$ & $\frac{2}{3}$ & $1-\frac{8}{3} \sin ^{2} \theta_{W}$ & -1 \\
$d, s, b$ & $-\frac{1}{3}$ & $-1+\frac{4}{3} \sin ^{2} \theta_{W}$ & 1 \\
\hline \hline
\end{tabular}




$$
i e \Gamma_{\mu}^{\gamma}
$$

para a interação eletromagnética e

$$
i \frac{g M_{Z}}{4 M_{W}}\left(\Gamma_{\mu}^{V}+\Gamma_{\mu}^{A}\right)
$$

para a interação fraca. As matrizes $\Gamma_{\mu}^{\gamma}, \Gamma_{\mu}^{V}$ e $\Gamma_{\mu}^{A}$ devem então carregar a informação de como o próton se acopla com um fóton e com as componentes vetorial e axial do campo do $Z$, respectivamente.

Dispondo agora de todos os elementos necessários, basta aplicar as regras de Feynman do Modelo Padrão [26] aos diagramas da Fig. 2.1 para obter as expressões das amplitudes relacionadas à interação eletromagnética,

$$
i \mathcal{M}_{\gamma}=\left[\bar{u}\left(k^{\prime}\right) i e e_{e} \gamma^{\mu} u(k)\right]\left(\frac{-i g_{\mu \nu}}{q^{2}}\right)\left[\bar{U}\left(p^{\prime}\right) i e \Gamma_{\gamma}^{\nu} U(p)\right]
$$

e à interação fraca,

$$
\begin{aligned}
i \mathcal{M}_{Z}= & {\left[\bar{u}\left(k^{\prime}\right) \frac{i g M_{Z}}{4 M_{W}} \gamma^{\mu}\left(g_{V}^{e}+g_{A}^{e} \gamma^{5}\right) u(k)\right]\left(\frac{-i g_{\mu \nu}}{q^{2}-M_{Z}^{2}}\right) } \\
& \times\left[\bar{U}\left(p^{\prime}\right) \frac{i g M_{Z}}{4 M_{W}}\left(\Gamma_{V}^{\nu}+\Gamma_{A}^{\nu}\right) U(p)\right],
\end{aligned}
$$

em que $k(p)$ e $k^{\prime}\left(p^{\prime}\right)$ são os momentos dos elétrons (prótons) incidente e espalhado e $q^{\prime} \equiv p^{\prime}-p=k-k^{\prime}$ é o momento trocado pelo bóson da interação. Definindo as correntes leptônicas $l$ e hadrônicas $J$ por

$$
\begin{aligned}
l^{\mu} & \equiv \bar{u}\left(k^{\prime}\right) \gamma^{\mu} u(k), \\
l^{\mu 5} & \equiv \bar{u}\left(k^{\prime}\right) \gamma^{\mu} \gamma^{5} u(k), \\
J_{\mu}^{\gamma} & \equiv \bar{U}\left(p^{\prime}\right) \Gamma_{\mu}^{\gamma} U(p), \\
J_{\mu}^{V} & \equiv \bar{U}\left(p^{\prime}\right) \Gamma_{\mu}^{V} U(p), \\
J_{\mu}^{A} & \equiv \bar{U}\left(p^{\prime}\right) \Gamma_{\mu}^{A} U(p),
\end{aligned}
$$

e considerando que $\frac{M_{Z}}{M_{W}} \approx 1$ e que $\left|q^{2}\right| \ll M_{Z}^{2}$ na região de momentos em que estamos interessados, escrevemos a expressão final para as amplitudes $\mathcal{M}_{\gamma}$ e $\mathcal{M}_{Z}$ : 


$$
\begin{aligned}
& \mathcal{M}_{\gamma}=\frac{4 \pi \alpha}{q^{2}} e_{e} l^{\mu} J_{\mu}^{\gamma}, \\
& \mathcal{M}_{Z}=-\frac{G_{F}}{2 \sqrt{2}}\left(g_{V}^{e} l^{\mu}+g_{A}^{e} l^{\mu 5}\right)\left(J_{\mu}^{V}+J_{\mu}^{A}\right),
\end{aligned}
$$

com $\alpha=\frac{e^{2}}{4 \pi}$ sendo a constante de estrutura fina e $G_{F}=\frac{g^{2}}{4 \sqrt{2} M_{W}^{2}}$ a constante de acoplamento de Fermi.

\subsection{Fatores de forma do próton}

Uma vez que as matrizes $\Gamma$ são introduzidas justamente porque o próton não é elementar, toda a informação sobre as suas propriedades eletromagnéticas e fracas devem estar contidas nas expressões analíticas de $\Gamma_{\mu}^{\gamma}, \Gamma_{\mu}^{V}$ e $\Gamma_{\mu}^{A}$. No entanto, seria extremamente difícil derivar estas expressões a partir de primeiros princípios, devido à complexidade da QCD a baixas energias. A solução para isto é parametrizar as matrizes usando funções escalares, dependentes do momento trocado $q^{2}$, chamadas de fatores de forma.

Nesta seção, faremos esta parametrização da maneira mais geral possível, tanto para a interação eletromagnética quanto para a interação fraca. Discutiremos em seguida a relação que existe entre os fatores de forma e as propriedades estáticas do próton, tais como distribuição de carga e magnetização. No final, mostraremos como é que cada sabor de quark contribui para os fatores de forma definidos.

\subsubsection{Interação Eletromagnética}

A corrente $J_{\mu}^{\gamma}$ definida na Eq. (2.9) é um quadrivetor, e para que a invariância de Lorentz seja mantida, $\Gamma_{\mu}^{\gamma}$ também deve ser. Por isso o anzatz mais geral é parametrizar $\Gamma_{\mu}^{\gamma} \mathrm{em}$ função dos quadrivetores disponíveis e das matrizes de Dirac $\gamma_{\mu}$. Assim,

$$
\Gamma_{\mu}^{\gamma}=\left[F_{1}^{\gamma p}\left(q^{2}\right) \gamma_{\mu}+\frac{i \sigma_{\mu \nu} q^{\nu}}{2 m_{N}} F_{2}^{\gamma p}\left(q^{2}\right)+F_{3}^{\gamma p}\left(q^{2}\right) q_{\mu}\right],
$$

em que $m_{N}$ é a massa do próton e $F_{1,2,3}^{\gamma p}$ são funções escalares que dependem do momento trocado $q^{2}$. Um termo proporcional a $\gamma_{5}$ não foi incluído porque a paridade é conservada nas interações eletromagnéticas. Também não incluímos o quadrivetor $\left(p+p^{\prime}\right)_{\mu}$ porque a matriz $\Gamma_{\mu}^{\gamma}$ é definida entre espinores e, neste caso, temos que

$$
\bar{U}\left(p^{\prime}\right)\left(p+p^{\prime}\right)_{\mu} U(p)=\bar{U}(p)\left[2 m_{N} \gamma_{\mu}-i \sigma_{\mu \nu} q^{\nu}\right] U(p)
$$


devido à identidade de Gordon (apresentada no Apêndice B). Desta forma, quando calculado entre espinores, o quadrivetor $\left(p+p^{\prime}\right)_{\mu}$ nada mais é do que uma combinação dos quadrivetores que acompanham as funções $F_{1}^{\gamma p}$ e $F_{2}^{\gamma p}$, e por isso não deve ser incluído numa parametrização geral.

O próximo passo para se obter a expressão de $\Gamma_{\mu}^{\gamma}$ é impor a conservação de corrente no vértice do próton. Desta imposição, deriva-se a identidade de Ward-Takahashi para o caso de um espalhamento elástico:

$$
\partial^{\mu} J_{\mu}^{\gamma} \Rightarrow q^{\mu} \Gamma_{\mu}^{\gamma} \rightarrow 0
$$

com $\Gamma_{\mu}^{\gamma}$ avaliada entre espinores. Vamos então verificar, termo a termo, se a expressão (2.14) respeita a condição em (2.16). Começando por $F_{1}^{\gamma p}$, temos

$$
\begin{aligned}
q^{\mu}\left[\bar{U}\left(p^{\prime}\right) F_{1}^{\gamma p}\left(q^{2}\right) \gamma_{\mu} U(p)\right] & =F_{1}^{\gamma p}\left(q^{2}\right) \bar{U}\left(p^{\prime}\right) \not q U(p) \\
& \left.=F_{1}^{\gamma p}\left(q^{2}\right) \bar{U}\left(p^{\prime}\right) \not \not p^{\prime}-\not p\right] U(p) \\
& =0 \forall F_{1}^{\gamma p}\left(q^{2}\right),
\end{aligned}
$$

em que usamos a equação de Dirac:

$$
\begin{gathered}
\not p U(p)=m_{N} U(p) \\
\bar{U}\left(p^{\prime}\right) \not p^{\prime}=\bar{U}\left(p^{\prime}\right) m_{N} .
\end{gathered}
$$

Agora, para o termo com $F_{2}^{\gamma p}$ :

$$
\begin{aligned}
q^{\mu}\left[\bar{U}\left(p^{\prime}\right) F_{2}^{\gamma p}\left(q^{2}\right) \sigma_{\mu \nu} q^{\nu} U(p)\right] & =\bar{U}\left(p^{\prime}\right) F_{2}^{\gamma p}\left(q^{2}\right)\left[\sigma_{\mu \nu} q^{\mu} q^{\nu}\right] U(p) \\
& =0 \forall F_{2}^{\gamma p}\left(q^{2}\right)
\end{aligned}
$$

pois a multiplicação entre o termo anti-simétrico $\sigma_{\mu \nu}$ e o simétrico $q^{\mu} q^{\nu}$ é zero. Por último, temos

$$
\begin{aligned}
q^{\mu}\left[\bar{U}\left(p^{\prime}\right) F_{3}^{\gamma p}\left(q^{2}\right) q_{\mu} U(p)\right] & =F_{3}^{\gamma p}\left(q^{2}\right) q^{2} \bar{U}\left(p^{\prime}\right) U(p) \\
& =0 \forall q^{2} \Leftrightarrow F_{3}^{\gamma p}\left(q^{2}\right)=0
\end{aligned}
$$

para o termo com $F_{3}^{\gamma p}$. Dos cálculos anteriores, concluímos que apenas $F_{1}^{\gamma p}\left(q^{2}\right)$ e $F_{2}^{\gamma p}\left(q^{2}\right)$ podem ser diferentes de zero, e finalmente chegamos à parametrização de $\Gamma_{\mu}^{\gamma}$ que conserva carga e paridade: 


$$
\Gamma_{\mu}^{\gamma}=\left[F_{1}^{\gamma p}\left(q^{2}\right) \gamma_{\mu}+\frac{i \sigma_{\mu \nu} q^{\nu}}{2 m_{N}} F_{2}^{\gamma p}\left(q^{2}\right)\right]
$$

em que as funções $F_{1}^{\gamma p}\left(q^{2}\right)$ e $F_{2}^{\gamma p}\left(q^{2}\right)$ são chamadas de fatores de forma de Dirac e Pauli, para a interação eletromagnética, respectivamente.

\subsubsection{Interação Fraca}

A corrente $J_{\mu}^{V}$, dada em $(2.10)$, descreve o acoplamento do próton com a componente vetorial do campo do $Z$, e por isso respeita as mesmas condições que $J_{\mu}^{\gamma}$ : conservação de carga e de paridade. Logo, toda a discussão da seção anterior também se aplica à matriz $\Gamma_{\mu}^{V}$, que deve ter uma expressão análoga à Eq. (2.19):

$$
\Gamma_{\mu}^{V}=\left[F_{1}^{Z p}\left(q^{2}\right) \gamma_{\mu}+\frac{i \sigma_{\mu \nu} q^{\nu}}{2 m_{N}} F_{2}^{Z p}\left(q^{2}\right)\right]
$$

Aqui temos novamente os fatores de forma de Dirac e Pauli, $F_{1}^{Z p}\left(q^{2}\right)$ e $F_{2}^{Z p}\left(q^{2}\right)$, mas para a interação fraca. No contexto do espalhamento elástico ep, existem de fato dois conjuntos destes fatores de forma, que são diferentes entre si.

Existe ainda uma corrente hadrônica adicional $J_{\mu}^{A}$, definida em (2.11), que leva em conta a componente axial da interação fraca. Claro que a conservação de carga também se aplica a $J_{\mu}^{A}$. No entanto, pela sua própria definição, esta corrente viola a paridade completamente, e por isso a matriz $\Gamma_{\mu}^{A}$ é parametrizada por:

$$
\Gamma_{\mu}^{A}=G_{A}^{Z p}\left(q^{2}\right) \gamma_{\mu} \gamma_{5}
$$

em que introduzimos o fator de forma axial do próton $G_{A}^{Z p}$. A corrente hadrônica fraca total é a soma das componentes vetorial e axial,

$$
\begin{aligned}
J_{\mu}^{Z} & \equiv J_{\mu}^{V}+J_{\mu}^{A} \\
& =\bar{U}\left(p^{\prime}\right)\left[\Gamma_{\mu}^{V}+\Gamma_{\mu}^{A}\right] U(p) \\
& =\bar{U}\left(p^{\prime}\right) \Gamma_{\mu}^{Z} U(p),
\end{aligned}
$$

em que se define, portanto,

$$
\Gamma_{\mu}^{Z}=\left[F_{1}^{Z p}\left(q^{2}\right) \gamma_{\mu}+\frac{i \sigma_{\mu \nu} q^{\nu}}{2 m_{N}} F_{2}^{Z p}\left(q^{2}\right)+G_{A}^{Z p} \gamma_{\mu} \gamma_{5}\right]
$$




\subsubsection{Fatores de forma e propriedades estáticas}

Seguindo uma analogia com a mecânica quântica não relativísica, poderíamos tentar interpretar os fatores de forma de Dirac e Pauli como sendo a transformada de Fourier das densidades de carga e de magnetização (elétrica e vetorial fraca) do próton. O problema é que uma distribuição espacial de carga deve necessariamente ser calculada a partir de uma transformada de Fourier tridimensional dos fatores de forma, e no caso relativístico estes fatores são uma função de $q^{2}=q_{0}^{2}-\vec{q}^{2}$, e não apenas do trivetor $\vec{q}$. Existe no entanto um referencial de Lorentz, chamado referencial de Breit, no qual a energia $q_{0}$ do bóson trocado se anula. É possível definir este referencial escolhendo, por exemplo, $\vec{p}^{\prime}=-\vec{p}=\frac{\vec{q}}{2}$.

Como vimos, os fatores de forma de Dirac e Pauli parametrizam as correntes $J_{\mu}^{\gamma}$ e $J_{\mu}^{V}$, que devido ao seu caráter vetorial têm a forma geral

$$
J_{\mu}=\bar{U}\left(p^{\prime}\right)\left[F_{1}^{p}\left(q^{2}\right) \gamma_{\mu}+\frac{i \sigma_{\mu \nu} q^{\nu}}{2 m_{N}} F_{2}^{p}\left(q^{2}\right)\right] U(p) .
$$

Pode ser demonstrado [27] que se definirmos os seguintes fatores de forma a partir das combinações de $F_{1,2}^{p}\left(q^{2}\right)$,

$$
\begin{aligned}
& G_{E}^{p}\left(q^{2}\right)=F_{1}^{p}\left(q^{2}\right)+\frac{q^{2}}{4 m_{N}^{2}} F_{2}^{p}\left(q^{2}\right), \\
& G_{M}^{p}\left(q^{2}\right)=F_{1}^{p}\left(q^{2}\right)+F_{2}^{p}\left(q^{2}\right),
\end{aligned}
$$

obtemos que a corrente hadrônica em (2.24), quando calculada no referencial de Breit, assume a seguinte estrutura:

$$
J_{\mu}^{\text {Breit }}=\left(G_{E}^{p}(\vec{q}), \frac{i(\vec{\sigma} \times \vec{q})}{2 m_{N}} G_{M}^{p}(\vec{q})\right) .
$$

O fator de forma $G_{E}^{p}(\vec{q})$ é então a própria componente temporal de $J_{\mu}^{\text {Breit }}$ e por isso é associado à transformada de Fourier da densidade de carga, enquanto que $G_{M}^{p}(\vec{q})$ aparece na componente espacial dentro de uma estrutura típica de momento magnético estático, e portanto é associado à transformada de Fourier da densidade de magnetização. Este novo conjunto de fatores de forma, chamados de fatores de forma de Sachs ou eletromagnéticos [28], fornecem então a relação entre a amplitude de espalhamento elástico $e p$ e as propriedades estáticas do próton.

É muito importante ressaltar que esta interpretação é válida exclusivamente no referencial de Breit. De fato, pensar em uma densidade espacial e estática de carga 
dentro do próton só faz sentido quando o tempo não entra na transformada de Fourier. A escolha do referencial de Breit é feita justamente para garantir esta condição.

Assim como acontece com $F_{1,2}^{p}\left(q^{2}\right)$, existem dois conjuntos diferentes de fatores de forma eletromagnéticos: $G_{E}^{\gamma p}\left(q^{2}\right)$ e $G_{M}^{\gamma p}\left(q^{2}\right)$, e os seus correspondentes na interação fraca, $G_{E}^{Z p}\left(q^{2}\right)$ e $G_{M}^{Z p}\left(q^{2}\right)$. Escolhemos a normalização destes fatores de forma com base na sua interpretação no referencial de Breit. No caso eletromagnético, usamos $G_{E}^{\gamma p}(0)=e_{p}=1$ e $G_{M}^{\gamma p}(0)=\mu_{p} \approx 2,79$ [29], e no caso fraco, $G_{E}^{Z p}(0)=g_{V}^{p}$ e $G_{M}^{Z p}(0)=\mu_{p}^{Z}$. Veja que isto já define também a normalização dos fatores de Pauli e Dirac.

\subsubsection{Decomposição dos fatores de forma nas contribuições dos quarks}

As propriedades eletromagnéticas e fracas do próton têm origem nos seus componentes fundamentais que sentem estas interações: os quarks. Até agora, definimos as correntes hadrônicas sem fazer nenhuma consideração a respeito da sua estrutura fundamental. No entanto, existe uma maneira de escrever as correntes (e portanto os fatores de forma) em função da contribuição dos quarks que compõem o próton.

No nível mais fundamental, um evento $e p \rightarrow e p$ ocorre por meio do espalhamento elástico entre o elétron e algum quark $q$ do próton. Em um processo deste tipo, as correntes do quark são análogas às correntes leptônicas em (2.7) e (2.8), pois os quarks são férmions fundamentais:

$$
\begin{gathered}
j_{q}^{\mu} \equiv \bar{u}_{q} \gamma^{\mu} u_{q}, \\
j_{q}^{\mu 5} \equiv \bar{u}_{q} \gamma^{\mu} \gamma^{5} u_{q},
\end{gathered}
$$

em que $u_{q}$ é o espinor do quark de sabor $q$. Em tal imagem do espalhamento, a amplitude $e p \rightarrow e p$ é então dada por uma média das seis possíveis amplitudes $e q \rightarrow e q$, poderada pelas cargas elétricas ou fracas, conforme o caso. Sendo assim, as correntes hadrônicas são dadas por [25]:

$$
\begin{aligned}
J_{\mu}^{\gamma} & \equiv\left\langle N\left(p^{\prime}\right)\left|\hat{J}_{\mu}^{\gamma}\right| N(p)\right\rangle \\
& =\left\langle N\left(p^{\prime}\right)\left|\sum_{q} e_{q} \bar{u}_{q} \gamma_{\mu} u_{q}\right| N(p)\right\rangle \\
& =\sum_{q} e_{q}\left\langle N\left(p^{\prime}\right)\left|\bar{u}_{q} \gamma_{\mu} u_{q}\right| N(p)\right\rangle
\end{aligned}
$$




$$
\begin{aligned}
& J_{\mu}^{V}=\sum_{q} g_{V}^{q}\left\langle N\left(p^{\prime}\right)\left|\bar{u}_{q} \gamma_{\mu} u_{q}\right| N(p)\right\rangle, \\
& J_{\mu}^{A}=\sum_{q} g_{A}^{q}\left\langle N\left(p^{\prime}\right)\left|\bar{u}_{q} \gamma_{\mu} \gamma_{5} u_{q}\right| N(p)\right\rangle,
\end{aligned}
$$

em que $e_{q}$ e $g_{V, A}^{q}$ são as cargas eletromagnéticas e fracas dos quarks, mostradas na Tabela 2.1. Nestas equações, as matrizes $\gamma_{\mu}$ e $\gamma_{5}$ são objetos bem definidos, mas o vetor de estado $|N\rangle$ do próton não é. Por isso, os elementos de matriz vetorial $\left\langle N\left(p^{\prime}\right)\left|\bar{u}_{q} \gamma_{\mu} u_{q}\right| N(p)\right\rangle$ e axial $\left\langle N\left(p^{\prime}\right)\left|\bar{u}_{q} \gamma_{\mu} \gamma_{5} u_{q}\right| N(p)\right\rangle$ também precisam ser parametrizados por fatores de forma. Novamente com base na conservação das correntes e considerações a respeito da paridade dos elementos de matriz, temos:

$$
\begin{aligned}
\left\langle N\left(p^{\prime}\right)\left|\bar{u}_{q} \gamma_{\mu} u_{q}\right| N(p)\right\rangle & =\bar{U}\left(p^{\prime}\right)\left[F_{1}^{q}\left(q^{2}\right) \gamma_{\mu}+\frac{i \sigma_{\mu \nu} q^{\nu}}{2 m_{N}} F_{2}^{q}\left(q^{2}\right)\right] U(p), \\
\left\langle N\left(p^{\prime}\right)\left|\bar{u}_{q} \gamma_{\mu} \gamma_{5} u_{q}\right| N(p)\right\rangle & =\bar{U}\left(p^{\prime}\right)\left[G_{A}^{q}\left(q^{2}\right) \gamma_{\mu} \gamma_{5}\right] U(p),
\end{aligned}
$$

em que $F_{1,2}^{q}\left(q^{2}\right)$ são os fatores de forma de Dirac e Pauli e $G_{A}^{q}\left(q^{2}\right)$ é o fator de forma axial, mas agora do quark de sabor $q$. Note que, ao contrário do que acontece no caso do próton, os fatores de forma $F_{1,2}^{q}$ não dependem do tipo de interação.

Para escrever as correntes hadrônicas em função da contribuição de cada um dos sabores, basta substituir as Eqs. (2.33) e (2.34) nas definições das correntes em (2.30) a (2.32). Deste modo, obtemos:

$$
\begin{aligned}
& J_{\mu}^{\gamma}= \sum_{q} e_{q} \bar{U}\left(p^{\prime}\right)\left[F_{1}^{q}\left(q^{2}\right) \gamma_{\mu}+\frac{i \sigma_{\mu \nu} q^{\nu}}{2 m_{N}} F_{2}^{q}\left(q^{2}\right)\right] U(p) \\
&= \bar{U}\left(p^{\prime}\right)\left[\left(\sum_{q} e_{q} F_{1}^{q}\left(q^{2}\right)\right) \gamma_{\mu}+\frac{i \sigma_{\mu \nu} q^{\nu}}{2 m_{N}}\left(\sum_{q} e_{q} F_{2}^{q}\left(q^{2}\right)\right)\right] U(p), \\
& J_{\mu}^{Z} \equiv J_{\mu}^{V}+J_{\mu}^{A} \\
&=\bar{U}\left(p^{\prime}\right)\left[\left(\sum_{q} g_{V}^{q} F_{1}^{q}\left(q^{2}\right)\right) \gamma_{\mu}+\frac{i \sigma_{\mu \nu} q^{\nu}}{2 m_{N}}\left(\sum_{q} g_{V}^{q} F_{2}^{q}\left(q^{2}\right)\right)\right. \\
&\left.+\left(\sum_{q} g_{A}^{q} G_{A}^{q}\left(q^{2}\right)\right) \gamma_{\mu} \gamma_{5}\right] U(p) .
\end{aligned}
$$


Comparando as Eqs. (2.35) e (2.36) com os resultados das seções anteriores — ou seja, definições das correntes hadrônicas em função das matrizes $\Gamma$ em (2.9) a (2.11) e a forma geral destas matrizes em (2.19) e (2.23) — podemos finalmente escrever os fatores de forma do próton como uma soma da contribuição dos seis sabores de quark:

$$
\begin{aligned}
& F_{1,2}^{\gamma p}=\sum_{q} e_{q} F_{1,2}^{q}, \\
& F_{1,2}^{Z p}=\sum_{q} g_{V}^{q} F_{1,2}^{q}, \\
& G_{A}^{Z p}=\sum_{q} g_{A}^{q} G_{A}^{q} .
\end{aligned}
$$

É claro que toda a discussão feita na seção 2.2.3 também se aplica no caso de $F_{1,2}^{q}$. Por isso convém definir os fatores de forma eletromagnéticos dos quarks,

$$
\begin{aligned}
& G_{E}^{q}\left(q^{2}\right)=F_{1}^{q}\left(q^{2}\right)+\frac{q^{2}}{4 m_{N}^{2}} F_{2}^{q}\left(q^{2}\right), \\
& G_{M}^{q}\left(q^{2}\right)=F_{1}^{q}\left(q^{2}\right)+F_{2}^{q}\left(q^{2}\right),
\end{aligned}
$$

que se relacionam com os fatores de forma elétromagnéticos e fracos do próton por

$$
\begin{aligned}
G_{E, M}^{\gamma p} & =\sum_{q} e_{q} G_{E, M}^{q}, \\
G_{E, M}^{Z p} & =\sum_{q} g_{V}^{q} G_{E, M}^{q} .
\end{aligned}
$$

Embora as somatórias nas Eqs. (2.39), (2.42) e (2.43) sejam sobre os seis sabores de quark, já é suficiente considerar apenas os três quarks mais leves $u, d$ e $s$. As massas dos três quarks mais pesados $c, b$ e $t$ são maiores do que a massa $m_{N}$ do próton, e por isso a contribuição destes sabores é fortemente suprimida [8]. Portanto, usando os valores das cargas dos quarks que constam na Tabela 2.1, 


$$
\begin{aligned}
G_{E, M}^{\gamma p}= & \frac{2}{3} G_{E, M}^{u}-\frac{1}{3} G_{E, M}^{d}-\frac{1}{3} G_{E, M}^{s}, \\
G_{E, M}^{Z p}= & \left(1-\frac{8}{3} \sin ^{2} \theta_{W}\right) G_{E, M}^{u}+\left(-1+\frac{4}{3} \sin ^{2} \theta_{W}\right) G_{E, M}^{d} \\
& +\left(-1+\frac{4}{3} \sin ^{2} \theta_{W}\right) G_{E, M}^{s}, \\
G_{A}^{Z p}= & -G_{A}^{u}+G_{A}^{d}+G_{A}^{s},
\end{aligned}
$$

em que $\theta_{W}$ denota o ângulo de Weinberg.

\subsection{Contribuição do quark estranho}

Faremos aqui um resumo de tudo o que foi visto na Seção 2.2, mas particularizando para o caso do quark $s$. Partindo da Eq. (2.33), temos que o acoplamento do fóton ou da parte vetorial do $Z$ com um quark $s$ de dentro do próton é dado pelo elemento de matriz:

$$
\left\langle N\left(p^{\prime}\right)\left|\bar{u}_{s} \gamma_{\mu} u_{s}\right| N(p)\right\rangle=\bar{U}\left(p^{\prime}\right)\left[F_{1}^{s}\left(q^{2}\right) \gamma_{\mu}+\frac{i \sigma_{\mu \nu} q^{\nu}}{2 m_{N}} F_{2}^{s}\left(q^{2}\right)\right] U(p),
$$

em que $F_{1}^{s}$ e $F_{2}^{s}$ são os fatores de forma estranhos de Dirac e Pauli. Os fatores de forma eletromagnéticos estranhos são definidos por

$$
\begin{aligned}
& G_{E}^{s}\left(q^{2}\right)=F_{1}^{s}\left(q^{2}\right)+\frac{q^{2}}{4 m_{N}^{2}} F_{2}^{s}\left(q^{2}\right), \\
& G_{M}^{s}\left(q^{2}\right)=F_{1}^{s}\left(q^{2}\right)+F_{2}^{s}\left(q^{2}\right),
\end{aligned}
$$

e quantificam a contribuição da estranheza para os fatores de forma de Sachs (eletromagnéticos e fracos) do próton. No referencial de Breit, no qual $q_{0}=0, G_{E}^{s}(\vec{q})$ e $G_{M}^{s}(\vec{q})$ podem ser interpretados como a transformada de Fourier das densidades de carga e de magnetização estranhas do próton, respectivamente. Desta forma, escolhemos a normalização $G_{E}^{s}(0)=0$, pois a carga estranha total do próton é nula, e $G_{M}^{s}(0)=\mu_{s}$, com $\mu_{s}$ sendo a contribuição do quark estranho para o momento magnético do próton. Veja que isso leva diretamente a $F_{1}^{s}(0)=0$ e $F_{2}^{s}(0)=G_{M}^{s}(0)=\mu_{s}$.

A corrente estranha pode também se acoplar com a componente axial do $Z$. Este acoplamento é descrito por 


$$
\left\langle N\left(p^{\prime}\right)\left|\bar{u}_{s} \gamma_{\mu} \gamma_{5} u_{s}\right| N(p)\right\rangle=\bar{U}\left(p^{\prime}\right)\left[G_{A}^{s}\left(q^{2}\right) \gamma_{\mu} \gamma_{5}\right] U(p),
$$

em que $G_{A}^{s}$ é o fator de forma estranho axial. Os dados experimentais que descrevemos neste trabalho são apenas dos fatores estranhos de Sachs, e não de $G_{A}^{s}$. No entanto, é conveniente defini-lo pois o seu valor medido é necessário para que se possa extrair $G_{E}^{s}$ e $G_{M}^{s}$ dos experimentos.

\subsection{Determinação experimental de $G_{E}^{s}$ e $G_{M}^{s}$}

Este trabalho de mestrado teve como objetivo descrever todos os dados experimentais existentes para $G_{E}^{s}$ e $G_{M}^{s}$, usando um modelo fenomenológico. Por isso, dedicamos esta seção à discussão de como estes fatores de forma se relacionam com grandezas acessíveis experimentalmente e qual o tipo de experimento em que eles são medidos. No final, também falaremos a respeito das colaborações que realizam tais experiências.

\subsection{1 $G_{E}^{s}$ e $G_{M}^{s}$ em função de observáveis}

A princípio. as Eqs. (2.44) e (2.45) poderiam indicar um meio de medir a contribuição da estranheza para os fatores de Sachs do próton. No entanto, lá aparecem cinco fatores de forma diferentes. Dois deles, $G_{E, M}^{\gamma p}$ e $G_{E, M}^{Z p}$, são acessíveis diretamente em experimentos e, portanto, conhecidos. Já os três restantes, $G_{E, M}^{u}, G_{E, M}^{d}$ e $G_{E, M}^{s}$, são quantidades desconhecidas. Logo, temos um sistema com duas equações e três variáveis, e então precisamos de mais uma equação para que os fatores de forma dos quarks, particulamente os do quark estranho, possam ser escritos em função de grandezas mensuráveis.

Uma forma de reduzir o número de variáveis é assumir a simetria de isospin. Em outras palavras, assumimos que a distribuição de quarks $u$ e $\bar{u}$ dentro de um próton seja exatamente igual à distribuição de quarks $d$ e $\bar{d}$ dentro de um neutron, e vice-versa. Além disso, assumimos também que a distribuição de estranheza dentro de qualquer nucleon seja sempre a mesma. Em resumo,

$$
p \rightarrow n \Rightarrow\left\{\begin{array}{c}
u \rightarrow d \\
d \rightarrow u \\
s \rightarrow s
\end{array}\right.
$$

Desta forma, em analogia com a Eq. (2.44), os fatores de Sachs do neutron em termos da contribuição dos quarks são: 


$$
G_{E, M}^{\gamma n}=\frac{2}{3} G_{E, M}^{d}-\frac{1}{3} G_{E, M}^{u}-\frac{1}{3} G_{E, M}^{s},
$$

Usando as Eqs. (2.44) e (2.51), podemos escrever:

$$
G_{E, M}^{\gamma p}-G_{E, M}^{\gamma n}=G_{E, M}^{u}-G_{E, M}^{d}
$$

Finalmente, usando as Eqs. (2.44) e (2.52), a expressão (2.45) se torna:

$$
G_{E, M}^{Z p}=\left(1-4 \sin ^{2} \theta_{W}\right) G_{E, M}^{\gamma p}-G_{E, M}^{\gamma n}-G_{E, M}^{s} .
$$

Esta expressão indica, portanto, um método de medida dos fatores de forma estranhos de Sachs. Os valores experimentais dos fatores de forma eletromagnéticos, tanto para o próton quanto para o neutron, são muito bem conhecidos (em particular o do próton, com grande precisão) [30]. Logo, $G_{E}^{s}$ e $G_{M}^{s}$ podem ser determinados por meio da medida dos fatores de forma fracos vetoriais. Estes últimos, por sua vez, são medidos em experimentos de violação de paridade em espalhamento elástico ep. Este é o assunto da próxima seção.

\subsubsection{Experimentos de violação de paridade em espalhamento}

$$
\text { ep } \rightarrow e p
$$

A seção de choque $e p \rightarrow e p$ contém, a princípio, as contribuições tanto da corrente eletromagnética quanto da corrente fraca. Entretanto, a interação eletromagnética é em muitas ordens de grandeza mais intensa do que a interação fraca quando $\left|q^{2}\right| \ll M_{Z}^{2}$. Para que os fatores de forma $G_{E, M}^{Z p}$ possam ser observados em espalhamento elástico ep, é necessário procurar grandezas físicas que evidenciem a contribuição fraca.

A violação de paridade é o que caracteriza a troca de um bóson $Z$. Por isso, se o objetivo é medir fatores de forma fracos, é interessante explorar observáveis que sejam conseqüência desta característica. Uma possibilidade é medir a assimetria, definida como

$$
A \equiv \frac{\sigma_{R}-\sigma_{L}}{\sigma_{R}+\sigma_{L}}
$$

em que $\sigma_{R}$ e $\sigma_{L}$ são as seções de choque $e p \rightarrow e p$ quando o elétron incidente tem helicidade positiva e negativa, respectivamente. Note que a assimetria pode assumir um valor diferente de zero justamente porque a interação fraca viola paridade, pois se a paridade fosse conservada teríamos $\sigma_{R}=\sigma_{L}$.

As seções de choque em (2.54) são calculadas, em primeira ordem, a partir das amplitudes definidas na Seção 2.1. Conforme está demonstrado na Ref. [25], a assimetria é então escrita como 


$$
A=\left(\frac{G_{F} q^{2}}{4 \sqrt{2} \pi \alpha}\right) \frac{\epsilon G_{E}^{\gamma p} G_{E}^{Z p}+\tau G_{M}^{\gamma p} G_{M}^{Z p}-\left(1-4 \sin ^{2} \theta_{W}\right) \epsilon^{\prime} G_{M}^{\gamma p} G_{A}^{Z p}}{\epsilon\left(G_{E}^{\gamma p}\right)^{2}+\tau\left(G_{M}^{\gamma p}\right)^{2}}
$$

em que

$$
\begin{aligned}
\tau & =-\frac{q^{2}}{4 m_{N}^{2}}, \\
\epsilon & =\frac{1}{1+2(1+\tau) \tan ^{2}(\theta / 2)}, \\
\epsilon^{\prime} & =\sqrt{\tau(1+\tau)\left(1-\epsilon^{2}\right)},
\end{aligned}
$$

são quantidades cinemáticas e $\theta$ é o ângulo de espalhamento do elétron no referencial do laboratório. Além de $G_{E, M}^{\gamma p}$, a assimetria depende também de $G_{A}^{Z p}$. Como não analisamos dados experimentais da contribuição do quark $s$ para a componente axial fraca, tal como ela é dada em (2.46), nos basta saber que $G_{A}^{Z p}$ é uma grandeza mensurável. De fato, a Colaboração SAMPLE a mediu num experimento de violação de paridade em espalhamento elétron-deuteron [31] e usou os resultados posteriormente para extrair dados de $G_{M}^{s}$.

Já que os valores experimentais $G_{A}^{Z p}, G_{E}^{\gamma p}$ e $G_{M}^{\gamma p}$ são conhecidos, a Eq. (2.55) nos dá um método eficiente para acessar os fatores de forma fracos. É ainda mais interessante, porém, que a assimetria seja escrita diretamente como função de $G_{E, M}^{s}$. Substituindo a Eq. (2.53) em (2.55), temos que

$$
\begin{aligned}
A= & \left(\frac{G_{F} q^{2}}{4 \sqrt{2} \pi \alpha}\right)\left\{\left[\left(1-4 \sin ^{2} \theta_{W}\right)-\frac{\epsilon G_{E}^{\gamma p} G_{E}^{\gamma n}+\tau G_{M}^{\gamma p} G_{M}^{\gamma n}}{\epsilon\left(G_{E}^{\gamma p}\right)^{2}+\tau\left(G_{M}^{\gamma p}\right)^{2}}\right]\right. \\
& \left.-\left[\frac{\epsilon G_{E}^{\gamma p} G_{E}^{s}+\tau G_{M}^{\gamma p} G_{M}^{s}}{\epsilon\left(G_{E}^{\gamma p}\right)^{2}+\tau\left(G_{M}^{\gamma p}\right)^{2}}\right]-\left(1-4 \sin ^{2} \theta_{W}\right) \epsilon^{\prime} G_{M}^{\gamma p} G_{A}^{Z p}\right\},
\end{aligned}
$$

ou ainda

$$
A=\left(\frac{G_{F} q^{2}}{4 \sqrt{2} \pi \alpha}\right)\left[A_{\gamma}+A_{s}+A_{A}\right]
$$

em que definimos as contribuições 


$$
\begin{aligned}
& A_{\gamma}=\left(1-4 \sin ^{2} \theta_{W}\right)-\frac{G_{E}^{\gamma n}+\eta G_{M}^{\gamma n}}{G_{E}^{\gamma p}+\eta G_{M}^{\gamma p}}, \\
& A_{s}=-\frac{G_{E}^{s}+\eta G_{M}^{s}}{G_{E}^{\gamma p}+\eta G_{M}^{\gamma p}}, \\
& A_{A}=-\left(1-4 \sin ^{2} \theta_{W}\right) \epsilon^{\prime} G_{M}^{\gamma p} G_{A}^{Z p},
\end{aligned}
$$

nas quais $\eta$ é dado por

$$
\eta\left(q^{2}\right)=\frac{\tau G_{M}^{\gamma p}\left(q^{2}\right)}{\epsilon G_{E}^{\gamma p}\left(q^{2}\right)}
$$

Desta discussão, concluímos que os fatores de forma estranhos de Sachs são de fato observados em medidas de assimetria, mas não de forma isolada, e sim dentro da combinação

$$
G_{E}^{s}\left(q^{2}\right)+\eta\left(q^{2}\right) G_{M}^{s}\left(q^{2}\right)
$$

Os primeiros dados experimentais para fatores de forma estranhos foram obtidos pelas colaborações SAMPLE [11-13] no MIT e HAPPEX [14-17] no TJNAF, para alguns valores de $Q^{2}=-q^{2}>0$ entre 0,1 e $0,5 \mathrm{GeV}^{2}$. A Colaboração HAPPEX mediu a assimetria para pequenos ângulos de espalhamento, e forneceu dados experimentais diretos para a combinação em (2.65), bem como dados de ajuste para $G_{E}^{s}$ e $G_{M}^{s}$. Já a Colaboração SAMPLE explorou uma outra região cinemática, com ângulos de espalhamento grandes, e assim pôde medir $G_{M}^{s}$ isoladamente. Note que isso acontece porque, de acordo com as definições em (2.57) e (2.64), $\eta$ assume valores cada vez maiores conforme o ângulo de espalhamento se aproxima de $180^{\circ}$ e assim, nesta região cinemática,

$$
\left|A_{s}\right| \approx \frac{G_{M}^{s}}{G_{M}^{\gamma p}}
$$

Mais tarde, outras duas colaborações fizeram medidas de assimetria na região de pequenos ângulos de espalhamento. A primeira foi a Colaboração A4 [18, 19] no MAMI, que além de medir $G_{E}^{s}+\eta G_{M}^{s}$ para $Q^{2}=0,109 \mathrm{GeV}^{2}$ e $Q^{2}=0,230 \mathrm{GeV}^{2}$, ainda utilizou resultados da SAMPLE para isolar alguns valores de $G_{E}^{s}$. Mais recentemente, a Colaboração G0 [20] no TJNAF forneceu uma grande quantidade de dados da combinação em (2.65) para uma região de momento transferido relativamente extensa: $0,12 \leq Q^{2} \leq$ $1,0 \mathrm{GeV}^{2}$. 
O único valor de $G_{E}^{s}$ que foi diretamente medido veio da Colaboração HAPPEX para $Q^{2}=0,091 \mathrm{GeV}^{2}$, mas de espalhamento de elétrons polarizados por um alvo de ${ }^{4} \mathrm{He}$ [32]. Neste caso específico, o espalhamento não depende de contribuições magnéticas e axiais, e a assimetria é dada por [25]

$$
A^{H e}=-\left(\frac{G_{F} q^{2}}{4 \sqrt{2} \pi \alpha}\right)\left[4 \sin ^{2} \theta_{W}+\frac{G_{E}^{s}}{G_{E}^{\gamma T=0}}\right],
$$

em que $G_{E}^{\gamma T=0}=\left(G_{E}^{\gamma p}+G_{E}^{\gamma n}\right) / 2$.

Uma última contribuição que usamos aqui foram os resultados de Pate et al. [23], no qual se fez uma análise global dos dados de $G_{E}^{s}+\eta G_{M}^{s}$ das colaborações G0 e HAPPEX em conjunto com os dados de $G_{A}^{Z p}$ da Colaboração E734 no BNL [24], resultando em dados separados de $G_{E}^{s}$ e $G_{M}^{s}$ para a região de momento $0,45<Q^{2}<1,0 \mathrm{GeV}^{2}$. 


\section{Capítulo 3}

\section{Modelo da nuvem mesônica}

A Eq. (2.47) nos mostra que uma expressão analítica para $F_{1}^{s}$ e $F_{2}^{s}$, e conseqüentemente para $G_{E}^{s}$ e $G_{M}^{s}$, pode ser obtida com o cálculo do elemento de matriz $\left\langle N\left(p^{\prime}\right)\left|\bar{s} \gamma_{\mu} s\right| N(p)\right\rangle$. A grande dificuldade é que o vetor de estado $|N(p)\rangle$ do próton não pode ser determinado a partir de primeiros princípios, já que a QCD não é perturbativa em energias hadrônicas. A solução é usar um modelo que, com base em considerações fenomenológicas, possa descrever $|N(p)\rangle$. Uma possibilidade é dada pelo modelo da nuvem mesônica (MCM). Neste capítulo, o objetivo é apresentar quantitativamente o MCM e estabelecer um método para determinar os fatores de forma estranhos usando este modelo.

\subsection{A nuvem de mésons estranhos}

A hipótese básica no modelo da nuvem é que o próton possui graus de liberdade internos de mésons e bárions. Mais especificamente, consideramos que um par quark-antiquark do mar de Dirac, durante o tempo permitido pelo princípio da incerteza, possa se acoplar com um dos quarks de valência, e desta maneira o próton é visto como um objeto que flutua em estados méson-bárion virtuais. Em particular, se um par $\bar{s} s$ é criado no mar, o próton pode então ser encontrado em um estado virtual constituído por um híperon e por um méson estranho com funções de onda dadas por $\left|q_{2} q_{3} s\right\rangle$ e $\left|q_{1} \bar{s}\right\rangle$, conforme está representado pictoricamente na Fig. 3.1.

Com base nessas considerações, o mecanismo básico do modelo é então representado pelo diagrama de Feynman da Fig. 3.2, e o vetor de estado do próton físico é dado pela soma do estado caroço que contém os três quarks de valência, mais uma série que envolve todos os estados virtuais possíveis [33], 


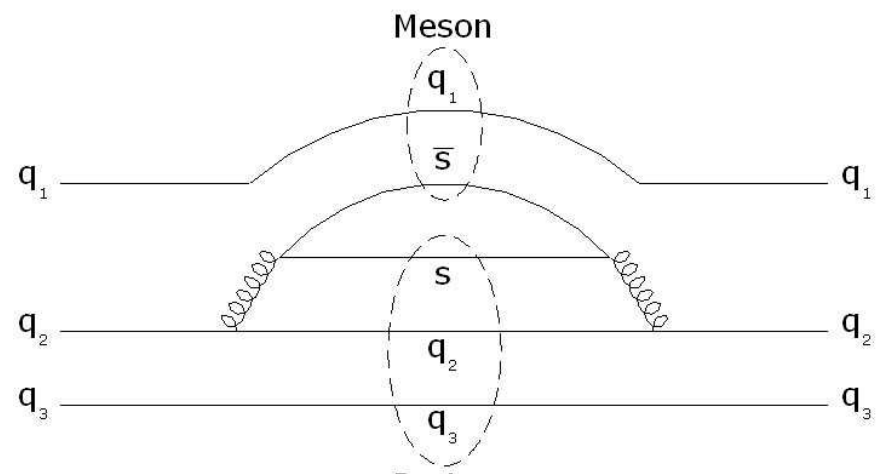

Barion

Figura 3.1: Representação pictórica do mecanismo do modelo da nuvem mesônica.

$$
|N(p)\rangle_{\text {físico }}=\sqrt{Z}\left\{|N(p)\rangle_{\text {caroço }}+\sum_{M, Y} \int d^{4} k g_{N M Y} \phi_{M, Y}(k)|M(k) ; Y(p-k)\rangle\right\}
$$

em que $M$ e $Y$ representam um méson estranho e um híperon, a função $\phi_{M, Y}$ é a amplitude de probabilidade de que o próton esteja em um estado formado por $M$ e $Y, \sqrt{Z}$ é a normalização do estado físico e também a probabilidade de que o próton esteja no estado caroço, $k$ denota o momento do méson e $g_{N M Y}$ é a constante de acoplamento do vértice próton-méson-bárion. A aproximação na Eq. (3.1) é válida enquanto a nuvem de mésons for relativamente leve $(Z \lesssim 1)$, de modo que não seja necessário incluir estados virtuais envolvendo híperons pesados $(\Xi, \Omega)$ acompanhados de dois ou três mésons.

A princípio, todos os hádrons formados por um quark ou antiquark $s$ poderiam ser incluídos na soma em (3.1), mas em geral se considera somente aqueles de menor massa, pois eles podem existir durante mais tempo e assim têm maior probabilidade de contribuir efetivamente nos experimentos que medem a estranheza do próton. Usamos aqui uma

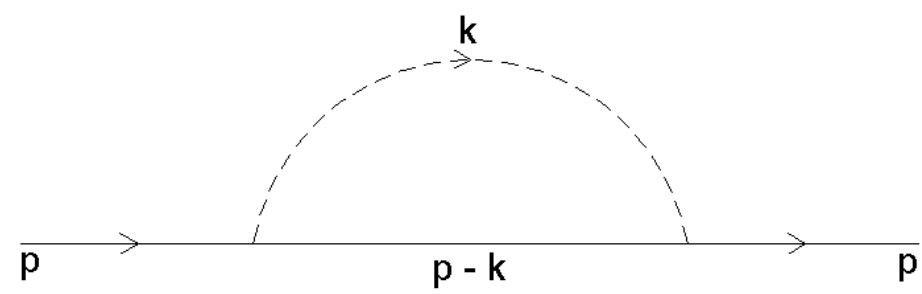

Figura 3.2: Diagrama de Feynman para o processo básico do modelo da nuvem mesônica. A linha tracejada representa um méson estranho $M(M=K, \kappa)$ e a linha contínua interna, um híperon $Y(Y=\Lambda, \Sigma)$. As linhas externas representam o próton. 
versão da nuvem que contém estados intermediários envolvendo os híperons $\Lambda$ e $\Sigma$ e os mésons estranhos $K$ e $\kappa$. Não incluímos o méson vetorial $K^{*}$ no modelo por razões já discutidas no Capítulo 1. A contribuição dos estados com o káon já havia sido determinada em um dos trabalhos do nosso grupo [21], de modo que calculamos somente os estados com o $\kappa$ e somamos os resultados com aqueles existentes para o káon.

\subsection{Calculando $G_{E}^{s}$ e $G_{M}^{s}$ no contexto da nuvem}

No cálculo de $\left\langle N\left(p^{\prime}\right)\left|\bar{s} \gamma_{\mu} s\right| N(p)\right\rangle$, representado pelo diagrama de Feynman da Fig. 3.3, não usaremos explicitamente o vetor de estado do próton, mas sim o processo básico do modelo da nuvem. De acordo com a Eq. (2.47), este elemento de matriz é dado por $\left\langle N\left(p^{\prime}\right)\left|\bar{s} \gamma_{\mu} s\right| N(p)\right\rangle=U\left(p^{\prime}\right) \Gamma_{\mu}^{s} U(p)$, em que definimos a matriz $\Gamma_{\mu}^{s}$ tal que

$$
U\left(p^{\prime}\right) \Gamma_{\mu}^{s} U(p)=\bar{U}\left(p^{\prime}\right)\left[F_{1}^{s}\left(q^{2}\right) \gamma_{\mu}+i \frac{\sigma_{\mu \nu} q^{\nu}}{2 m_{N}} F_{2}^{s}\left(q^{2}\right)\right] U(p)
$$

quando avaliada entre espinores. Para um próton descrito pelo MCM, o diagrama da Fig. 3.3 deve ser igual a uma soma de diagramas de loop análogos ao da Fig. 3.2, porém com um fóton acoplado a cada um deles. Assim, $\Gamma_{\mu}^{s}$ será igual à soma das amplitudes de Feynman relacionadas com estes loops, ou ainda, igual à amplitude de Feynman total associada ao processo representado na Fig. 3.3. Para calcular as amplitudes dos loops, é necessário estabelecer dois pontos importantes. O primeiro deles é definir a lagrangiana de interação do vértice $N \kappa Y$. O segundo, é descobrir como que um fóton se acopla com o próton quando este último é descrito no contexto da nuvem. Comecemos com a lagrangiana.

Como no modelo da nuvem se assume que os graus de liberdade internos do próton são hadrônicos, os vértices devem ser descritos por lagrangianas efetivas, que na nossa versão da nuvem são [34]

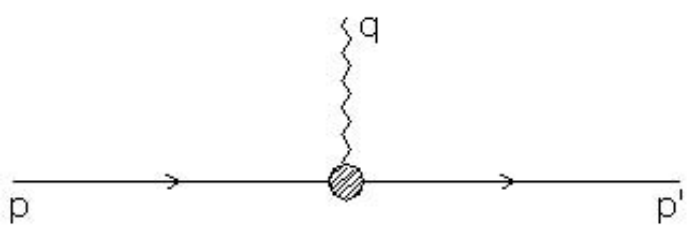

Figura 3.3: Diagrama de Feynman que representa o acoplamento do fóton com a componente estranha do próton. 


$$
\begin{aligned}
& \mathcal{L}_{N \kappa \Lambda}=-g_{N \kappa \Lambda} \bar{\Psi}_{N} \Psi_{\Lambda} \phi_{\kappa}, \\
& \mathcal{L}_{N \kappa \Sigma}=-g_{N \kappa \Sigma} \bar{\Psi}_{N}\left(\vec{\tau} \cdot \vec{\Psi}_{\Sigma}\right) \phi_{\kappa},
\end{aligned}
$$

para estados intermediários com o méson $\kappa$. O campo fermiônico do próton $(\Lambda, \Sigma)$ é denotado por $\Psi_{N(\Lambda, \Sigma)}$ e $\phi_{\kappa}$ é o campo escalar do $\kappa$. A menos de índices de isospin, as lagrangianas em (3.3) e (3.4) são análogas, de modo que não há necessidade de explicitar o cálculo para os dois híperons. Daqui em diante faremos as contas somente para o caso do $\Lambda$ e mostraremos como incluir a contribuição do $\Sigma$ na Seção 3.4.

A lagrangiana na Eq. (3.3) descreve apenas a interação entre partículas pontuais, já que não contém nenhum termo que leve em conta a estrutura interna e o tamanho dos hádrons envolvidos. Um procedimento comum neste caso é incluir um fator de forma efetivo na constante de acoplamento. Nessa versão do MCM, incluímos o fator de forma usado no potencial de Bonn-Jülich para interação híperon-nucleon [35],

$$
F\left(k^{2}\right)=\frac{m_{\kappa}^{2}-\Lambda_{\kappa}^{2}}{k^{2}-\Lambda_{\kappa}^{2}},
$$

que tem a forma convencional de monopólo e, embora devesse depender de todos os momentos envolvidos no processo, é parametrizado somente em função do momento $k$ do méson. Na Eq. (3.5), $m_{\kappa}$ é a massa do $\kappa$ e $\Lambda_{\kappa}$ é o cut-off, que trata-se de um parâmetro de ajuste. O fator de forma é introduzido no cálculo por meio da substituição

$$
g_{N \kappa \Lambda} \rightarrow g_{N \kappa \Lambda} F\left(k^{2}\right) .
$$

Veja que esse tipo de parametrização leva a dois limites físicos que, de fato, devem existir na teoria. Primeiro, $F\left(k^{2}\right)=1$ para $\Lambda \rightarrow \infty$, e neste limite a lagrangiana reproduz a interação entre partículas pontuais. Além disso, para interação entre partículas extensas $\left(\Lambda_{\kappa}\right.$ finito), temos $F\left(k^{2}\right) \rightarrow 0$ quando $k^{2}$ assume valores muito grandes, o que suprime a flutuação do próton em estados méson-bárion neste limite.

Note porém que a introdução de um fator de forma dependente de $k^{2}$ na constante de acoplamento também cria um problema. Isto faz com que a lagrangiana depedenda da derivada comum $\partial_{x}$, já que esta corresponde ao momento $k$ no espaço das posições, e isso quebra a invariância de gauge da lagrangiana. Para que a invariância de gauge seja reestabelecida é necessário que a derivada comum seja substituída pela derivada covariante

$$
\partial_{x}^{\mu} \rightarrow \partial_{x}^{\mu}-i Q_{\kappa} A(x)^{\mu}
$$


em que $A(x)$ representa o campo do fóton. Este procedimento, também conhecido como substituição mínima, traz conseqüências ao nosso segundo ponto de discussão, sobre como o fóton se acopla com o próton do MCM. Abordaremos este assunto em profundidade na seção a seguir.

\subsection{Acoplamento do fóton com o próton do MCM}

A consideração mais simples que poderíamos fazer seria assumir que o acoplamento fótonpróton se dá pelo acoplamento do fóton com o méson e com o híperon, considerados pontuais pois a inclusão de $F\left(k^{2}\right)$ no vértice já dá conta do tamanho de todos os hádrons envolvidos. Mas veja que, neste caso, chegaríamos a uma amplitude total que obviamente não é invariante de gauge, pois a troca da derivada comum pela covariante na lagrangiana já introduz por si só o acoplamento do fóton com o vértice extenso $N \kappa \Lambda$.

Nesta seção iremos além desta argumentação qualitativa e demostraremos que, de fato, os acoplamentos pontuais com os estados virtuais não são suficientes para construir uma amplitude total que obedeça a invariância de gauge, mas que esta última pode ser reestabelecida se adicionarmos o acoplamento com o vértice.

\subsubsection{Acoplamentos pontuais com os estados virtuais}

Como dissemos, a estrutura interna do híperon e do méson virtuais já foi considerada com a inclusão do fator de forma $F\left(k^{2}\right)$ no vértice $N \kappa \Lambda$; por isso, no nosso modelo, estes hádrons são vistos simplesmente como partículas pontuais que carregam carga estranha. Sendo assim, os acoplamentos do fóton com o híperon e o méson, representados pelos diagramas (a) e (b) da Fig. 3.4, podem ser obtidos diretamente das regras de Feynman da QED [26]:

$$
\begin{aligned}
& \left\langle\Lambda\left(p^{\prime}\right)\left|\bar{s} \gamma_{\mu} s\right| \Lambda(p)\right\rangle=Q_{\Lambda} \bar{U}\left(p^{\prime}\right) \gamma_{\mu} U(p), \\
& \left\langle\kappa\left(p^{\prime}\right)\left|\bar{s} \gamma_{\mu} s\right| \kappa(p)\right\rangle=Q_{\kappa}\left(p+p^{\prime}\right)_{\mu}
\end{aligned}
$$

com $Q_{\Lambda}=-1$ sendo a carga estranha do híperon e $Q_{\kappa}=1$, a carga estranha do méson $\kappa$. Aplicando as regras de Feynman aos diagramas, obtemos as amplitudes associadas a cada um dos processos, 


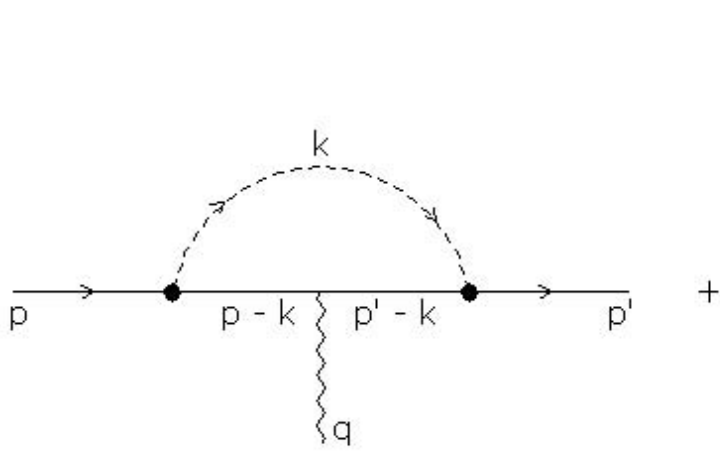

(a)

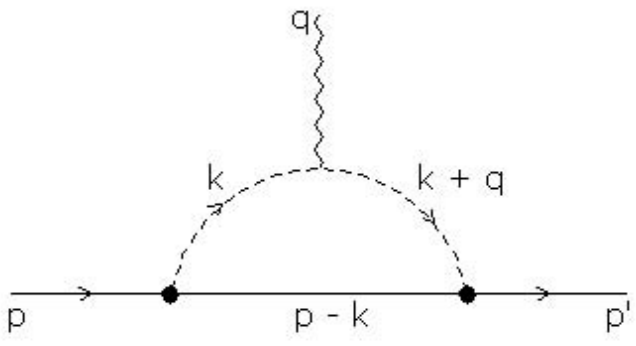

(b)

Figura 3.4: Diagramas de Feynman para o acoplamento do fóton com o híperon (a) e com o méson (b) virtuais. As linhas contínuas interna e externa representam o híperon e o próton, respectivamente. A linha pontilhada representa o méson $\kappa$.

$$
\begin{aligned}
\Gamma_{\mu}^{\Lambda}\left(p, p^{\prime}\right)= & -g_{N \kappa \Lambda}^{2} Q_{\Lambda} \int \frac{d^{4} k}{(2 \pi)^{4}} F^{2}\left(k^{2}\right) \Delta_{\kappa}\left(k^{2}\right) S_{\Lambda}\left(p^{\prime}-k\right) \gamma_{\mu} S_{\Lambda}(p-k), \\
\Gamma_{\mu}^{\kappa}\left(p, p^{\prime}\right)= & -g_{N \kappa \Lambda}^{2} Q_{\kappa} \int \frac{d^{4} k}{(2 \pi)^{4}} F\left(k^{2}\right) F\left((k+q)^{2}\right)(2 k+q)_{\mu} \\
& \left.\times \Delta_{\kappa}\left(k^{2}\right) \Delta_{\kappa}\left((k+q)^{2}\right)\right) S_{\Lambda}(p-k),
\end{aligned}
$$

em que os propagadores do méson e do híperon são

$$
\begin{aligned}
\Delta_{\kappa}\left(k^{2}\right) & =\frac{i}{k^{2}-m_{\kappa}^{2}+i \epsilon}, \\
S_{Y}(p) & =\frac{i\left(\not p+m_{Y}\right)}{p^{2}-m_{Y}^{2}+i \epsilon},
\end{aligned}
$$

respectivamente, com $m_{Y}$ sendo a massa do híperon e, no caso deste cálculo, $Y=\Lambda$. Se estes dois acoplamentos já fossem suficientes para construir uma amplitude de Feynman invariante de gauge, então a identidade de Ward-Takahashi (WT) deveria ser válida para $\Gamma_{\mu}^{\Lambda}+\Gamma_{\mu}^{\kappa}$. No caso de diagramas de um loop, a identidade WT é dada por [36]

$$
q^{\mu} \Gamma_{\mu}\left(p, p^{\prime}\right)=Q\left(\Sigma(p)-\Sigma\left(p^{\prime}\right)\right)
$$

em que $Q \equiv Q_{\Lambda}+Q_{\kappa}$ é a carga estranha do próton $(Q=0$, evidentemente), e $\Sigma(p)$ é a auto-energia do processo representado na Fig. 3.2, escrita como 


$$
\Sigma(p)=-i g_{N \kappa \Lambda}^{2} \int \frac{d^{4} k}{(2 \pi)^{4}} F^{2}\left(k^{2}\right) \Delta_{\kappa}\left(k^{2}\right) S_{\Lambda}(p-k),
$$

ou ainda, com a mudança de variável $k \rightarrow p-k$,

$$
\Sigma(p)=-i g_{N \kappa \Lambda}^{2} \int \frac{d^{4} k}{(2 \pi)^{4}} F^{2}\left((p-k)^{2}\right) \Delta_{\kappa}\left((p-k)^{2}\right) S_{\Lambda}(k) .
$$

Verifiquemos então a validade da Eq. (3.14) começando com o acoplamento da corrente com o híperon. Multiplicando a Eq. (3.10) por $q^{\mu}$, temos

$$
q^{\mu} \Gamma_{\mu}^{\Lambda}=-g_{N \kappa \Lambda}^{2} Q_{\Lambda} \int \frac{d^{4} k}{(2 \pi)^{4}} F^{2}\left(k^{2}\right) \Delta_{\kappa}\left(k^{2}\right) S_{\Lambda}\left(p^{\prime}-k\right) \not q S_{\Lambda}(p-k) .
$$

Da definição do propagador do híperon em (3.13) e usando $q=p^{\prime}-p$, obtemos a identidade

$$
S_{\Lambda}\left(p^{\prime}-k\right) \not q S_{\Lambda}(p-k)=i\left(S_{\Lambda}(p-k)-S_{\Lambda}\left(p^{\prime}-k\right)\right),
$$

cuja demonstração está feita no Apêndice A. Substituindo (3.18) em (3.17) e comparando com as expressões (3.15) e (3.16) para a auto-energia, temos diretamente

$$
q^{\mu} \Gamma_{\mu}^{\Lambda}=Q_{\Lambda}\left(\Sigma(p)-\Sigma\left(p^{\prime}\right)\right)
$$

mostrando que a identidade WT é válida para o caso bariônico. Já no caso do acoplamento com o méson, multiplicando a Eq. (3.11) por $q^{\mu}$ podemos escrever

$$
\begin{aligned}
q^{\mu} \Gamma_{\mu}^{\kappa}= & -g_{N \kappa \Lambda}^{2} Q_{\kappa} \int \frac{d^{4} k}{(2 \pi)^{4}} F\left(k^{2}\right) F\left((k+q)^{2}\right)\left(2 k \cdot q+q^{2}\right) \\
& \left.\times \Delta_{\kappa}\left(k^{2}\right) \Delta_{\kappa}\left((k+q)^{2}\right)\right) S_{\Lambda}(p-k) .
\end{aligned}
$$

Da definição do propagador do $\kappa$, dada em (3.12), facilmente se demonstra a identidade

$$
\left(2 k \cdot q+q^{2}\right) \Delta_{\kappa}\left(k^{2}\right) \Delta_{\kappa}\left((k+q)^{2}\right)=i\left[\Delta_{\kappa}\left(k^{2}\right)-\Delta_{\kappa}\left((k+q)^{2}\right)\right] .
$$

Substituindo (3.21) em (3.20), obtemos

$$
\begin{aligned}
q^{\mu} \Gamma_{\mu}^{\kappa}= & -i g_{N \kappa \Lambda}^{2} Q_{\kappa} \int \frac{d^{4} k}{(2 \pi)^{4}}\left[F\left(k^{2}\right) F\left((k+q)^{2}\right) \Delta_{\kappa}\left(k^{2}\right) S_{\Lambda}(p-k)\right. \\
& \left.-F\left(k^{2}\right) F\left((k+q)^{2}\right) \Delta_{\kappa}\left((k+q)^{2}\right) S_{\Lambda}(p-k)\right] .
\end{aligned}
$$


Com a mudança de variável $k \rightarrow p-k$ no segundo termo da integral em (3.22) chegamos à equação

$$
\begin{aligned}
q^{\mu} \Gamma_{\mu}^{\kappa}= & -i g_{N \kappa \Lambda}^{2} Q_{\kappa} \int \frac{d^{4} k}{(2 \pi)^{4}}\left[F\left(k^{2}\right) F\left((k+q)^{2}\right) \Delta_{\kappa}\left(k^{2}\right) S_{\Lambda}(p-k)\right. \\
& \left.-F\left((p-k)^{2}\right) F\left(\left(p^{\prime}-k\right)^{2}\right) \Delta_{\kappa}\left(\left(p^{\prime}-k\right)^{2}\right) S_{\Lambda}(k)\right]
\end{aligned}
$$

que quando comparada às expressões (3.15) e (3.16) para a auto-energia, implica que

$$
q^{\mu} \Gamma_{\mu}^{\kappa} \neq Q_{\kappa}\left(\Sigma(p)-\Sigma\left(p^{\prime}\right)\right)
$$

e, portanto, que a identidade WT não se verifica no caso mesônico. Com os resultados em (3.19) e (3.24) concluímos que uma amplitude de Feynman dada pela soma $\Gamma_{\mu}^{\kappa}+\Gamma_{\mu}^{\Lambda}$ não obedece à identidade WT. Veja que isso só acontece por causa da inclusão do fator de forma: tomando $F\left(k^{2}\right)=1$ na Eq. (3.23) chegaríamos diretamente a $q^{\mu} \Gamma_{\mu}^{\kappa}=$ $Q_{\kappa}\left(\Sigma(p)-\Sigma\left(p^{\prime}\right)\right)$.

\subsubsection{Função de vértice seagull}

O próximo passo agora seria incluir o acoplamento do fóton com o vértice não pontual $N \kappa \Lambda$. Acontece que ainda não sabemos como descrever este acoplamento, ou seja, não conhecemos a função de um vértice que inclui os três hádrons mais um fóton, também chamado de vértice seagull. Para deduzir a função de vértice não basta substituir a derivada covariante em $\mathcal{L}_{N \kappa \Lambda}$, pois a dependência da lagrangiana com a derivada comum é quadrática, o que torna o cálculo muito mais complexo. Faremos aqui a dedução desta função seguindo o método desenvolvido por Ohta [37].

Partindo da substituição em (3.6), temos para a interação $N \kappa \Lambda$ a seguinte função de vértice:

$$
\Gamma\left(k^{2}\right)=-g_{N \kappa \Lambda} F\left(k^{2}\right) .
$$

Para que possamos incluir o campo do fóton via substituição mínima, primeiro é preciso expandir $F\left(k^{2}\right)$ em uma série de potências:

$$
\Gamma\left(k^{2}\right)=-g_{N \kappa \Lambda} \sum_{l} c_{l} k^{2 l}
$$

Em seguida, aplicamos a transformada de Fourier na Eq. (3.26). Assim, 


$$
\begin{aligned}
\Gamma(x, y) & =\int \frac{d^{4} k}{(2 \pi)^{4}} \Gamma\left(k^{2}\right) e^{i k \cdot(x-y)} \\
& =-\frac{g_{N \kappa \Lambda}}{(2 \pi)^{4}} \sum_{l} c_{l} \int d^{4} k k^{2 l} e^{i k \cdot(x-y)} \\
& =-\frac{g_{N \kappa \Lambda}}{(2 \pi)^{4}} \sum_{l} c_{l}(i)^{2 l}\left(\partial_{x}\right)^{2 l} \int d^{4} k e^{i k \cdot(x-y)} \\
& =-g_{N \kappa \Lambda} \sum_{l} c_{l}(-1)^{l}\left(\partial_{x}\right)^{2 l} \delta^{4}(y-x) .
\end{aligned}
$$

Usando agora a expressão (3.7) na equação acima obtemos a função de vértice modificada pela inclusão do campo do fóton,

$$
\tilde{\Gamma}(x, y)=-g_{N \kappa \Lambda} \sum_{l} c_{l}(-1)^{l}\left[\partial_{x}-i Q_{\kappa} A(x)\right]^{2 l} \delta^{4}(y-x)
$$

na qual o termo entre colchetes admite a expansão

$$
\begin{aligned}
{\left[\partial_{x}-i Q_{\kappa} A(x)\right]^{2 l}=} & \partial_{x}^{2 l}-i Q_{\kappa}\left[\partial_{x}^{2(l-1)}\left(\partial_{x} \cdot A(x)+A(x) \cdot \partial_{x}\right)\right. \\
& +\partial_{x}^{2(l-2)}\left(\partial_{x} \cdot A(x)+A(x) \cdot \partial_{x}\right) \partial_{x}^{2}+\ldots \\
& \left.+\left(\partial_{x} \cdot A(x)+A(x) \cdot \partial_{x}\right) \partial_{x}^{2(l-1)}\right]+\mathcal{O}\left(A^{2}\right)
\end{aligned}
$$

Por causa da delta de Dirac em (3.28), os $\partial_{x}$ 's da direita de $A_{\mu}(x)$ na expansão podem ser substituídos por $-\partial_{y}$ 's. Logo,

$$
\begin{aligned}
{\left[\partial_{x}-i Q_{\kappa} A(x)\right]^{2 l} \rightarrow } & \partial_{x}^{2 l}-i Q_{\kappa}\left(\partial_{x}-\partial_{y}\right)^{\mu} \\
& \times\left[\partial_{x}^{2(l-1)}+\partial_{x}^{2(l-2)} \partial_{y}^{2}+\cdots+\partial_{y}^{2(l-1)}\right] A_{\mu}(x)+\mathcal{O}\left(A^{2}\right)
\end{aligned}
$$

Ao substituirmos (3.30) em (3.28), as derivadas parciais em $x$ estarão agindo simultaneamente no campo do fóton e na delta de Dirac. Para separar a diferenciação da delta e do campo, é conveniente reescrever $A_{\mu}(x)$ na forma

$$
A_{\mu}(x)=\int d^{4} z A_{\mu}(z) \delta^{4}(z-x)
$$

de maneira que a expressão para a função do vértice $N \kappa \Lambda$ modificada seja 


$$
\begin{aligned}
\tilde{\Gamma}(x, y)= & -g_{N \kappa \Lambda} \sum_{l} c_{l}(-1)^{l}\left(\partial_{x}\right)^{2 l} \delta^{4}(y-x) \\
& +i g_{N \kappa \Lambda} Q_{\kappa}\left(\partial_{x}-\partial_{y}\right)^{\mu} \sum_{l} c_{l}(-1)^{l}\left[\partial_{x}^{2(l-1)}+\partial_{x}^{2(l-2)} \partial_{y}^{2}+\cdots+\partial_{y}^{2(l-1)}\right] \\
& \times \int d^{4} z A_{\mu}(z) \delta^{4}(z-x) \delta^{4}(y-x)+\mathcal{O}\left(A^{2}\right) .
\end{aligned}
$$

Note que agora a função $\tilde{\Gamma}(x, y)$ está na forma da expansão em série

$$
\tilde{\Gamma}(x, y)=\Gamma(x, y)+\int d^{4} z \Delta \Gamma^{\mu}(x, y, z) A_{\mu}(z)+\mathcal{O}\left(A^{2}\right),
$$

da qual coletamos a amplitude $\Delta \Gamma^{\mu}$ proporcional a $A_{\mu}(z)$ porque estamos interessados no acoplamento de um único fóton com o próton,

$$
\begin{aligned}
\Delta \Gamma_{\mu}(x, y, z)= & i g_{N \kappa \Lambda} Q_{\kappa}\left(\partial_{x}-\partial_{y}\right)_{\mu} \sum_{l} c_{l}(-1)^{l} \\
& \times\left[\partial_{x}^{2(l-1)}+\partial_{x}^{2(l-2)} \partial_{y}^{2}+\cdots+\partial_{y}^{2(l-1)}\right] \delta^{4}(z-x) \delta^{4}(y-x) .
\end{aligned}
$$

O próximo passo é susbstituir a forma integral da delta de Dirac na equação acima. Além disso, para escrever os termos entre colchetes numa forma mais compacta, vamos introduzir a função de dois operadores arbitrários $a$ e $b$,

$$
\Phi_{l}(a, b)=a^{l-1}+a^{l-2} b+\cdots+b^{l-1}=a^{l-1}\left[\frac{(b / a)^{l}-1}{(b / a)-1}\right]=\frac{a^{l}-b^{l}}{a-b}
$$

de modo que

$$
\begin{aligned}
\Delta \Gamma_{\mu}(x, y, z)= & i g_{N \kappa \Lambda} Q_{\kappa} \int \frac{d^{4} k d^{4} q}{(2 \pi)^{8}}\left(\partial_{x}-\partial_{y}\right)_{\mu} \\
& \times \sum_{l} c_{l}(-1)^{l} \Phi_{l}\left(\partial_{x}^{2}, \partial_{y}^{2}\right) e^{-i q \cdot(z-x)} e^{-i k \cdot(y-x)}
\end{aligned}
$$

Aplicando as derivadas no argumento das exponenciais e também a identidade

$$
(-1)^{l} \Phi_{l}\left(-(k+q)^{2},-k^{2}\right)=-\Phi_{l}\left((k+q)^{2}, k^{2}\right)
$$

obtemos a expressão para a amplitude do vértice de interação com o fóton, mas ainda no espaço das configurações, 


$$
\begin{aligned}
\Delta \Gamma_{\mu}(x, y, z)= & g_{N \kappa \Lambda} Q_{\kappa} \int \frac{d^{4} k d^{4} q}{(2 \pi)^{8}}(q+2 k)_{\mu} \\
& \times \sum_{l} c_{l} \Phi_{l}\left((k+q)^{2}, k^{2}\right) e^{i k \cdot(x-y)} e^{i q \cdot(x-z)} .
\end{aligned}
$$

Na Ref. [37], a transformada de Fourier está definida por

$$
\Delta \Gamma_{\mu}(x, y, z)=\int \frac{d^{4} k d^{4} q}{(2 \pi)^{8}} \Delta \Gamma_{\mu}(k, q) e^{i k \cdot(y-x)} e^{i q \cdot(x-z)}
$$

Portanto, por simples comparação entre (3.38) e (3.39), obtemos a amplitude no espaço dos momentos,

$$
\Delta \Gamma_{\mu}(k, q)=g_{N \kappa \Lambda} Q_{\kappa}(q-2 k)_{\mu} \sum_{l} c_{l} \Phi_{l}\left((k-q)^{2}, k^{2}\right)
$$

que pela definição de $\Phi_{l}$ em (3.35) pode ser reescrita como

$$
\Delta \Gamma_{\mu}(k, q)=-g_{N \kappa \Lambda} Q_{\kappa} \frac{(q-2 k)_{\mu}}{(k-q)^{2}-k^{2}}\left[\sum_{l} c_{l} k^{2 l}-\sum_{l} c_{l}(k-q)^{2 l}\right]
$$

Note que na expressão acima as somatórias podem ser identificadas com a própria expansão do fator de forma. De maneira geral, escrevemos

$$
i \Delta \Gamma_{\mu}(k, q)= \pm i g_{N \kappa \Lambda} Q_{\kappa}(q \pm 2 k)_{\mu} \frac{F\left(k^{2}\right)-F\left((k \pm q)^{2}\right)}{(k \pm q)^{2}-k^{2}}
$$

em que os sinais superior e inferior estão associados a um méson entrando e saindo do vértice, respectivamente.

\subsubsection{Acoplamento com o vértice não pontual}

Uma vez que deduzimos a função de vértice seagull (3.42), vamos calcular a contribuição do acoplamento com o vértice extenso $N \kappa \Lambda$, representado pelos diagramas (a) e (b) da Fig. 3.5. Aplicando as regras de Feynman aos diagramas, temos 


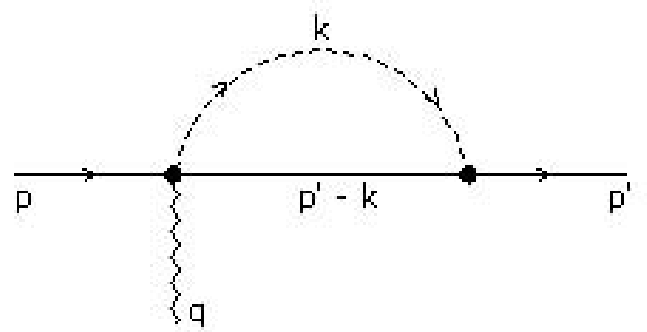

(a)

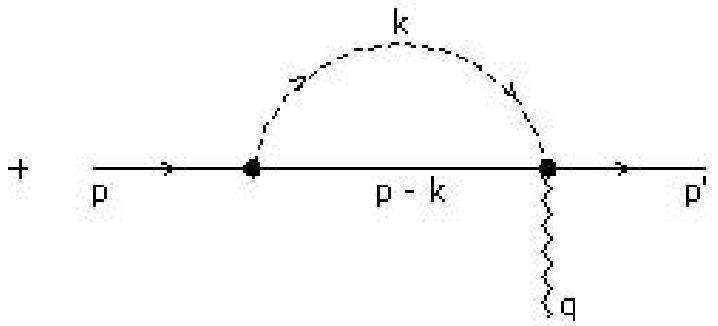

(b)

Figura 3.5: Idem à Fig. 3.4, mas agora considerando o acoplamento do fóton com o vértice extenso $N \kappa \Lambda$.

$$
\begin{aligned}
\Gamma_{\mu}^{v 1}\left(p, p^{\prime}\right)= & i g_{N \kappa \Lambda}^{2} Q_{\kappa} \int \frac{d^{4} k}{(2 \pi)^{4}} \frac{(q-2 k)_{\mu}}{(k-q)^{2}-k^{2}} \\
& \times \Delta_{\kappa}\left(k^{2}\right) S_{\Lambda}\left(p^{\prime}-k\right) F\left(k^{2}\right)\left[F\left(k^{2}\right)-F\left((k-q)^{2}\right)\right], \\
\Gamma_{\mu}^{v 2}\left(p, p^{\prime}\right)= & -i g_{N \kappa \Lambda}^{2} Q_{\kappa} \int \frac{d^{4} k}{(2 \pi)^{4}} \frac{(q+2 k)_{\mu}}{(k+q)^{2}-k^{2}} \\
& \times \Delta_{\kappa}\left(k^{2}\right) S_{\Lambda}(p-k) F\left(k^{2}\right)\left[F\left(k^{2}\right)-F\left((k+q)^{2}\right)\right] .
\end{aligned}
$$

em que $\Gamma_{\mu}^{v 1}$ e $\Gamma_{\mu}^{v 2}$ são as amplitudes associadas aos diagramas (a) e (b), respectivamente. Essas amplitudes já nos dão a contribuição do acoplamento fóton-vértice para a amplitude total $\Gamma_{\mu}^{\Lambda}+\Gamma_{\mu}^{\kappa}+\Gamma_{\mu}^{v 1}+\Gamma_{\mu}^{v 2}$, mas ainda falta verificar se esta soma realmente é invariante de gauge. Multiplicando as Eqs. (3.43) e (3.44) por $q^{\mu}$ e usando a igualdade

$$
q^{\mu} \frac{(q \pm 2 k)_{\mu}}{(k \pm q)^{2}-k^{2}}=1
$$

segue que

$$
\begin{aligned}
q^{\mu} \Gamma_{\mu}^{v}= & i g_{N \kappa \Lambda}^{2} Q_{\kappa} \int \frac{d^{4} k}{(2 \pi)^{4}}\left\{\Delta_{\kappa}\left(k^{2}\right) S_{\Lambda}\left(p^{\prime}-k\right) F\left(k^{2}\right)\left[F\left(k^{2}\right)-F\left((k-q)^{2}\right)\right]\right. \\
& \left.-\Delta_{\kappa}\left(k^{2}\right) S_{\Lambda}(p-k) F\left(k^{2}\right)\left[F\left(k^{2}\right)-F\left((k+q)^{2}\right)\right]\right\}
\end{aligned}
$$

com $\Gamma_{\mu}^{v}=\Gamma_{\mu}^{v 1}+\Gamma_{\mu}^{v 2}$. Fazendo a substituição $k \rightarrow p^{\prime}-k$ no primeiro termo da integral e lembrando que $q=p^{\prime}-p$, reescrevemos (3.46) como 


$$
\begin{aligned}
q^{\mu} \Gamma_{\mu}^{v}= & -i g_{N \kappa \Lambda}^{2} Q_{\kappa} \int \frac{d^{4} k}{(2 \pi)^{4}} \\
& \left\{\left[F^{2}\left(k^{2}\right) \Delta_{\kappa}\left(k^{2}\right) S_{\Lambda}(p-k)-F^{2}\left(\left(p^{\prime}-k\right)^{2}\right) \Delta_{\kappa}\left(\left(p^{\prime}-k\right)^{2}\right) S_{\Lambda}(k)\right]\right. \\
& +\left[F\left(k^{2}\right) F\left((k+q)^{2}\right) \Delta_{\kappa}\left(k^{2}\right) S_{\Lambda}(p-k)\right. \\
& \left.\left.-F\left((p-k)^{2}\right) F\left(\left(p^{\prime}-k\right)^{2}\right) \Delta_{\kappa}\left(\left(p^{\prime}-k\right)^{2}\right) S_{\Lambda}(k)\right]\right\} .
\end{aligned}
$$

Por simples comparação dos termos da integral em (3.47) com as Eqs. (3.15), (3.16) e (3.23), obtemos

$$
q^{\mu} \Gamma_{\mu}^{v}=Q_{\kappa}\left(\Sigma(p)-\Sigma\left(p^{\prime}\right)\right)-q^{\mu} \Gamma_{\mu}^{\kappa}
$$

Finalmente, lembrando que a identidade WT é válida para o acoplamento bariônico, somamos (3.19) com (3.48) para provar que a amplitude $\Gamma_{\mu}^{\Lambda}+\Gamma_{\mu}^{\kappa}+\Gamma_{\mu}^{v}$ é invariante de gauge, ou seja,

$$
q^{\mu}\left(\Gamma_{\mu}^{\Lambda}+\Gamma_{\mu}^{\kappa}+\Gamma_{\mu}^{v}\right)=\left(Q_{\Lambda}+Q_{\kappa}\right)\left(\Sigma(p)-\Sigma\left(p^{\prime}\right)\right)
$$

\subsection{Incluindo a contribuição do $\Sigma$}

A única diferença entre as lagrangianas em (3.3) e (3.4) são os índices de isospin que aparecem no segundo caso. No contexto da nuvem mesônica, a lagrangiana $\mathcal{L}_{N \kappa \Sigma}$ nos diz que existem dois estados virtuais possíveis quando o híperon é um $\Sigma$ : ou o próton flutua no estado $\left|\kappa^{+} ; \Sigma^{0}\right\rangle$, ou então, no estado $\left|\kappa^{0} ; \Sigma^{+}\right\rangle$. Para cada uma destas possibilidades temos diagramas de Feynman idênticos àqueles das Figs. 3.4 e 3.5.

Em termos das regras de Feynman, os vértices $N \kappa^{+} \Sigma^{0}$ e $N \kappa^{0} \Sigma^{+}$irão contribuir com

$$
\begin{aligned}
& -i g_{N \kappa \Sigma}\left\langle N \mid \kappa^{+} ; \Sigma^{0}\right\rangle_{\text {isospin }} \\
& -i g_{N \kappa \Sigma}\left\langle N \mid \kappa^{0} ; \Sigma^{+}\right\rangle_{\text {isospin }}
\end{aligned}
$$

conforme o caso. Os elementos de matriz nas Eqs. (3.50) e (3.51) pertencem ao espaço de isospin e modulam as amplitudes associadas aos processos $p \rightarrow \kappa^{+} \Sigma^{0}$ e $p \rightarrow \kappa^{0} \Sigma^{+}$. Conhecendo o isospin (e as projeções) de cada hádron envolvido e usando os coeficientes de Clebsch-Gordan [29], obtemos 


$$
\begin{aligned}
& \left\langle N \mid \kappa^{+} ; \Sigma^{0}\right\rangle_{\text {isospin }}=\left\langle\frac{1}{2} \frac{1}{2} \mid \frac{1}{2} \frac{1}{2} ; 10\right\rangle=-\sqrt{\frac{1}{3}} \\
& \left\langle N \mid \kappa^{0} ; \Sigma^{+}\right\rangle_{\text {isospin }}=\left\langle\frac{1}{2} \frac{1}{2} \mid \frac{1}{2}-\frac{1}{2} ; 11\right\rangle=\sqrt{\frac{2}{3}} .
\end{aligned}
$$

Aplicando as regras de Feynman aos diagramas das Figs. 3.4 e 3.5 com o híperon virtual sendo um $\Sigma$, devemos obter expressões análogas às Eqs. (3.10), (3.11), (3.43) e (3.44), mas neste caso moduladas pelo quadrado dos elementos de matriz em (3.52) e (3.53), uma vez que cada diagrama de loop contém dois vértices $N \kappa \Sigma$. Assim, a soma das contribuições dos estados virtuais $\left|\kappa^{+} ; \Sigma^{0}\right\rangle$ e $\left|\kappa^{0} ; \Sigma^{+}\right\rangle$resulta em

$$
\begin{aligned}
\Gamma_{\mu}^{\Sigma}\left(p, p^{\prime}\right)= & -g_{N \kappa \Sigma}^{2} Q_{\Sigma} \mathcal{I} \int \frac{d^{4} k}{(2 \pi)^{4}} F^{2}\left(k^{2}\right) \Delta_{\kappa}\left(k^{2}\right) S_{\Sigma}\left(p^{\prime}-k\right) \gamma_{\mu} S_{\Sigma}(p-k), \\
\Gamma_{\mu}^{\kappa}\left(p, p^{\prime}\right)= & -g_{N \kappa \Sigma}^{2} Q_{\kappa} \mathcal{I} \int \frac{d^{4} k}{(2 \pi)^{4}} F\left(k^{2}\right) F\left((k+q)^{2}\right)(2 k+q)_{\mu} \\
& \left.\times \Delta_{\kappa}\left(k^{2}\right) \Delta_{\kappa}\left((k+q)^{2}\right)\right) S_{\Sigma}(p-k), \\
\Gamma_{\mu}^{v 1}\left(p, p^{\prime}\right)= & i g_{N \kappa \Sigma}^{2} Q_{\kappa} \mathcal{I} \int \frac{d^{4} k}{(2 \pi)^{4}} \frac{(q-2 k)_{\mu}}{(k-q)^{2}-k^{2}} \\
& \times \Delta_{\kappa}\left(k^{2}\right) S_{\Sigma}\left(p^{\prime}-k\right) F\left(k^{2}\right)\left[F\left(k^{2}\right)-F\left((k-q)^{2}\right)\right], \\
\Gamma_{\mu}^{v 2}\left(p, p^{\prime}\right)= & -i g_{N \kappa \Sigma}^{2} Q_{\kappa} \mathcal{I} \int \frac{d^{4} k}{(2 \pi)^{4}} \frac{(q+2 k)_{\mu}}{(k+q)^{2}-k^{2}} \\
& \times \Delta_{\kappa}\left(k^{2}\right) S_{\Sigma}(p-k) F\left(k^{2}\right)\left[F\left(k^{2}\right)-F\left((k+q)^{2}\right)\right] .
\end{aligned}
$$

em que $\mathcal{I}=\left|\left\langle N \mid \kappa^{+} ; \Sigma^{0}\right\rangle_{\text {isospin }}\right|^{2}+\left|\left\langle N \mid \kappa^{0} ; \Sigma^{+}\right\rangle_{\text {isospin }}\right|^{2}$. De acordo com as Eqs. (3.52) e (3.53) temos $\mathcal{I}=1$, o que elimina a dependência da amplitude com o isospin. Logo, a contribuição dos estados com o $\Sigma$, considerando todas as combinações de isospin possíveis, é exatamente igual a dos estados com o $\Lambda$. 


\subsection{Amplitude total do processo}

Sumarizando os resultados mais importantes deste capítulo, vimos que o diagrama de Feynman da Fig. 3.3 que descreve o acoplamento entre o fóton e a estranheza do próton é dado pela soma de diagramas de loop, conforme está representado na Fig. 3.6, quando o próton é descrito pelo modelo da nuvem mesônica. A amplitude de Feynman total, que provamos ser invariante de gauge, é a soma de todos os processos,

$$
\Gamma_{\mu}^{s}=\sum_{Y=\Lambda, \Sigma}\left[\Gamma_{\mu}^{Y}+\Gamma_{\mu}^{\kappa}+\Gamma_{\mu}^{v 1}+\Gamma_{\mu}^{v 2}\right]
$$

em que as contribuições do híperon, do méson e do vértice são
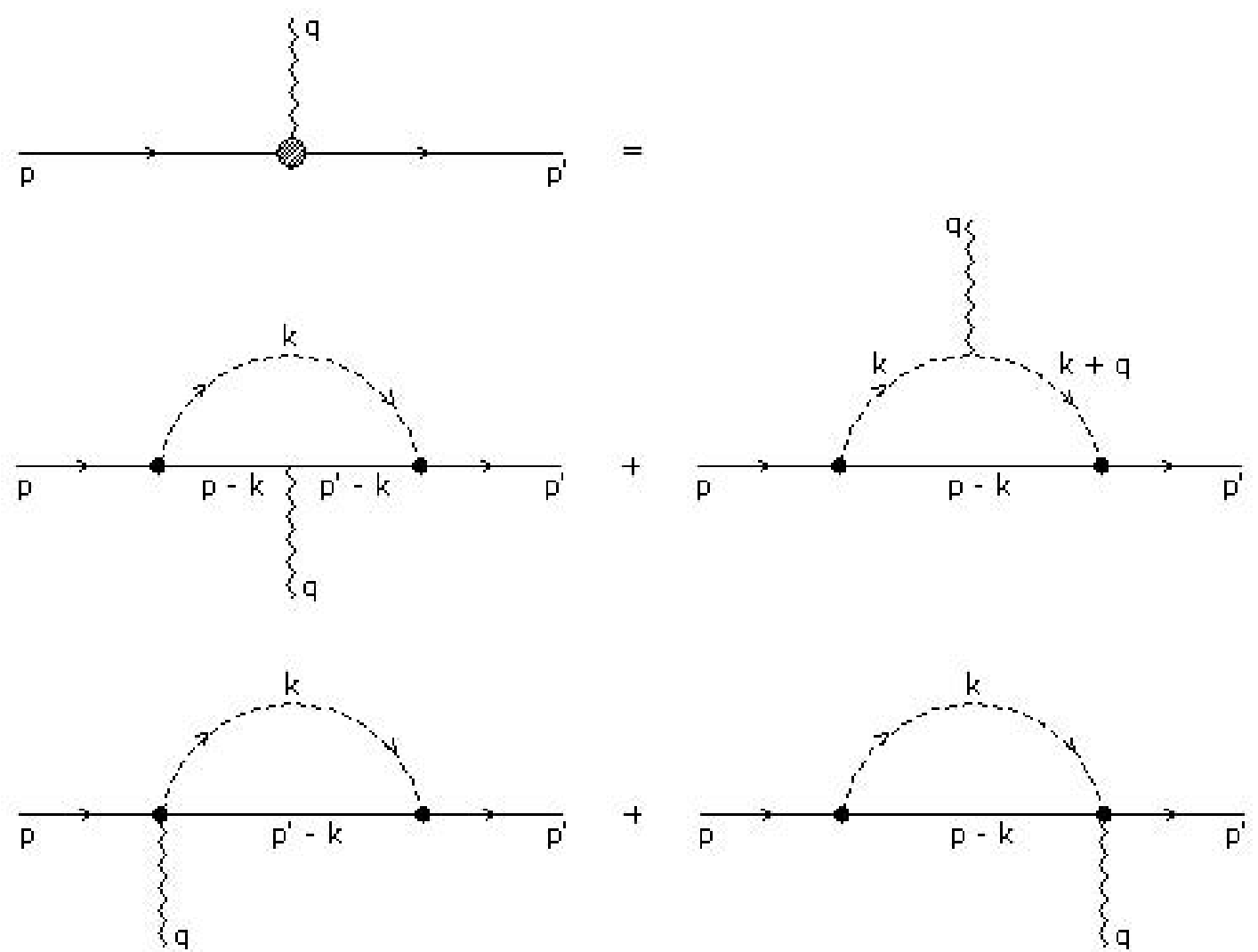

Figura 3.6: Diagramas de Feynman que contribuem para a amplitude total $\Gamma_{\mu}^{s}$ invariante de gauge. As linhas contínuas interna e externa representam um híperon $Y(Y=\Lambda, \Sigma)$ e um próton, respectivamente. A linha pontilhada representa o méson $\kappa$. 


$$
\begin{aligned}
\Gamma_{\mu}^{Y}\left(p, p^{\prime}\right)= & -g_{N \kappa Y}^{2} Q_{Y} \int \frac{d^{4} k}{(2 \pi)^{4}} F^{2}\left(k^{2}\right) \Delta_{\kappa}\left(k^{2}\right) S_{\Lambda}\left(p^{\prime}-k\right) \gamma_{\mu} S_{\Lambda}(p-k), \\
\Gamma_{\mu}^{\kappa}\left(p, p^{\prime}\right)= & -g_{N \kappa Y}^{2} Q_{\kappa} \int \frac{d^{4} k}{(2 \pi)^{4}} F\left(k^{2}\right) F\left((k+q)^{2}\right)(2 k+q)_{\mu} \\
& \left.\times \Delta_{\kappa}\left(k^{2}\right) \Delta_{\kappa}\left((k+q)^{2}\right)\right) S_{\Lambda}(p-k), \\
\Gamma_{\mu}^{v 1}\left(p, p^{\prime}\right)= & i g_{N \kappa Y}^{2} Q_{\kappa} \int \frac{d^{4} k}{(2 \pi)^{4}} \frac{(q-2 k)_{\mu}}{(k-q)^{2}-k^{2}} \\
& \times \Delta_{\kappa}\left(k^{2}\right) S_{\Lambda}\left(p^{\prime}-k\right) F\left(k^{2}\right)\left[F\left(k^{2}\right)-F\left((k-q)^{2}\right)\right], \\
\Gamma_{\mu}^{v 2}\left(p, p^{\prime}\right)= & -i g_{N \kappa Y}^{2} Q_{\kappa} \int \frac{d^{4} k}{(2 \pi)^{4}} \frac{(q+2 k)_{\mu}}{(k+q)^{2}-k^{2}} \\
& \times \Delta_{\kappa}\left(k^{2}\right) S_{\Lambda}(p-k) F\left(k^{2}\right)\left[F\left(k^{2}\right)-F\left((k+q)^{2}\right)\right],
\end{aligned}
$$

respectivamente. A Eq. (3.2) relaciona a amplitude total $\Gamma_{\mu}^{s} \operatorname{com} F_{1}^{s}$ e $F_{2}^{s}$, o que estabelece um método efetivo para se determinar os fatores de forma estranhos. O próximo passo do nosso cálculo analítico, e também o último, será desenvolver as integrais das Eqs. (3.59) a (3.62) de maneira que seja possível isolar $F_{1}^{s}$ e $F_{2}^{s}$ e, a partir destes, obter $G_{E}^{s}$ e $G_{M}^{s}$. 


\section{Capítulo 4}

\section{Cálculo dos fatores de forma estranhos}

Neste capítulo mostraremos como extrair os fatores de forma a partir das amplitudes que obtivemos no Capítulo 3. O método para desenvolver as expressões das amplitudes é semelhante para todos os acoplamentos, de forma que detalharemos o cálculo somente para o caso bariônico.

\subsection{Acoplamento bariônico}

Substituindo as expressões dos propagadores e do fator de forma $F\left(k^{2}\right)$ na Eq. (3.59), escrevemos $\Gamma_{\mu}^{Y}$ como

$$
\begin{aligned}
\Gamma_{\mu}^{Y}\left(p, p^{\prime}\right) & =i g_{N \kappa Y}^{2} Q_{Y}\left(m_{\kappa}^{2}-\Lambda_{\kappa}^{2}\right)^{2} \int \frac{d^{4} k}{(2 \pi)^{4}}\left(\not p^{\prime}-\not k+m_{Y}\right) \gamma_{\mu}\left(\not p-\not k+m_{Y}\right) \\
& \times \frac{1}{\left(k^{2}-\Lambda_{\kappa}^{2}\right)^{2}\left(k^{2}-m_{\kappa}^{2}\right)\left[\left(p^{\prime}-k\right)^{2}-m_{Y}^{2}\right]\left[(p-k)^{2}-m_{Y}^{2}\right]} .
\end{aligned}
$$

Lembrando que a amplitude é sempre avaliada entre espinores, desenvolvemos a parte matricial da equação anterior usando a equação de Dirac, dada em (2.17), de modo que

$$
\left(\not p^{\prime}-\not k+m_{Y}\right) \gamma_{\mu}\left(\not p-\not k+m_{Y}\right) \rightarrow\left[\left(m_{Y}+m_{N}\right)^{2}-k^{2}\right] \gamma_{\mu}-2\left(m_{Y}+m_{N}\right) k_{\mu}+2 \not k k_{\mu} .
$$

A integral na variável $k$ pode ser resolvida mais facilmente se escrevermos o termo com o denominador na forma de uma exponencial. Isto pode ser feito com a parametrização de Schwinger, que é dada na Eq. (B.1) do apêndice. 
Depois de usarmos a equação de Dirac e a fórmula de Schwinger, temos que a amplitude é dada por

$$
\begin{aligned}
\Gamma_{\mu}^{Y}\left(p, p^{\prime}\right)= & -i g_{N \kappa Y}^{2} Q_{Y}\left(m_{\kappa}^{2}-\Lambda_{\kappa}^{2}\right)^{2} \int_{0}^{\infty} \prod_{i=1}^{4} d \alpha_{i} \alpha_{1} \exp C \\
& \times \int \frac{d^{4} k}{(2 \pi)^{4}} \exp \left(A_{4} k^{2}+2 B \cdot k\right)\left[\left(\delta^{2}-k^{2}\right) \gamma_{\mu}-2 \delta k_{\mu}+2 \not k k_{\mu}\right],
\end{aligned}
$$

em que definimos

$$
\begin{aligned}
\delta & =m_{Y}+m_{N}, \\
\delta_{2} & =m_{Y}^{2}-m_{N}^{2}, \\
A_{4} & =\sum_{i=1}^{4} \alpha_{i} \\
B & =-\alpha_{3} p^{\prime}-\alpha_{4} p, \\
C & =-\alpha_{1} \Lambda_{\kappa}^{2}-\alpha_{2} m_{\kappa}^{2}-\left(\alpha_{3}+\alpha_{4}\right) \delta_{2} .
\end{aligned}
$$

Na expressão (4.3) temos integrais quadridimensionais em $k$, nas quais a exponencial está multiplicada por $k_{\mu}, k^{2}=g^{\mu \nu} k_{\mu} k_{\nu}$ e $k k_{\mu}=\gamma^{\nu} k_{\mu} k_{\nu}$. Usando as expressões (B.3) a (B.5) do apêndice para resolver estas integrais, obtemos:

$$
\begin{aligned}
\Gamma_{\mu}^{Y}\left(p, p^{\prime}\right)= & \frac{g_{N \kappa Y}^{2}}{(4 \pi)^{2}} Q_{Y}\left(m_{\kappa}^{2}-\Lambda_{\kappa}^{2}\right)^{2} \int_{0}^{\infty} \prod_{i=1}^{4} d \alpha_{i} \frac{\alpha_{1}}{A_{4}^{2}} \exp \left(C-\frac{B^{2}}{A_{4}}\right) \\
& \times\left[\delta^{2} \gamma_{\mu}+\frac{\gamma_{\mu}+2 \delta B_{\mu}}{A_{4}}+\frac{2 \not B B_{\mu}-\gamma_{\mu} B^{2}}{A_{4}^{2}}\right] .
\end{aligned}
$$

O objetivo agora é abrir a expressão de $\Gamma_{\mu}^{Y}$ e separar os termos proporcionais a $\left(p+p^{\prime}\right)$, de maneira que possamos usar a identidade de Gordon, dada na Eq. (B.2) do apêndice. Substituindo as definições de $B$ e $C$ e usando que $B^{2}=\left(\alpha_{3}+\alpha_{4}\right)^{2} m_{N}^{2}-\alpha_{3} \alpha_{4} q^{2}$, reescrevemos a amplitude como 


$$
\begin{aligned}
\Gamma_{\mu}^{Y}\left(p, p^{\prime}\right)= & \frac{g_{N \kappa Y}^{2}}{(4 \pi)^{2}} Q_{Y}\left(m_{\kappa}^{2}-\Lambda_{\kappa}^{2}\right)^{2} \int_{0}^{\infty} \prod_{i=1}^{4} d \alpha_{i} \frac{\alpha_{1}}{A_{4}^{2}} \\
& \exp \left\{-\alpha_{1} \Lambda_{\kappa}^{2}-\alpha_{2} m_{\kappa}^{2}-\left(\alpha_{3}+\alpha_{4}\right) \delta_{2}-\frac{\left(\alpha_{3}+\alpha_{4}\right)^{2} m_{N}^{2}-\alpha_{3} \alpha_{4} q^{2}}{A_{4}}\right\} \\
& \times\left[\left(\delta^{2}+\frac{1}{A_{4}}-\frac{\left(\alpha_{3}+\alpha_{4}\right)^{2} m_{N}^{2}-\alpha_{3} \alpha_{4} q^{2}}{A_{4}^{2}}\right) \gamma_{\mu}-\frac{2 \delta\left(\alpha_{3} p^{\prime}+\alpha_{4} p\right)_{\mu}}{A_{4}}\right. \\
& \left.+\frac{2\left(\alpha_{3} \not p^{\prime}+\alpha_{4} \not \not\right)\left(\alpha_{3} p^{\prime}+\alpha_{4} p\right)_{\mu}}{A_{4}^{2}}\right] .
\end{aligned}
$$

Veja que o integrando da equação anterior é simétrico pela troca $\alpha_{3} \leftrightarrow \alpha_{4}$, e por isso os termos proporcionais a $\left(\alpha_{3}-\alpha_{4}\right)$ se anulam. Logo,

$$
\left(\alpha_{3} p^{\prime}+\alpha_{4} p\right)_{\mu} \rightarrow\left(\frac{\alpha_{3}+\alpha_{4}}{2}\right)\left(p^{\prime}+p\right)_{\mu}
$$

Considerando novamente que a amplitude é calculada entre espinores de Dirac, temos ainda que

$$
\left(\alpha_{3} \not p^{\prime}+\alpha_{4} \not p\right) \rightarrow\left(\frac{\alpha_{3}+\alpha_{4}}{2}\right)\left(\not p^{\prime}+\not p\right) \rightarrow\left(\alpha_{3}+\alpha_{4}\right) m_{N}
$$

e assim,

$$
\begin{aligned}
\Gamma_{\mu}^{Y}\left(p, p^{\prime}\right)= & c\left(\Lambda_{\kappa}^{2}\right) Q_{Y} \int_{0}^{\infty} \prod_{i=1}^{4} d \alpha_{i} \frac{\alpha_{1}}{A_{4}^{2}} \exp \left(-f_{b}\left(\alpha_{i}, q^{2}\right)\right) \\
& \left\{\left[\delta^{2}+\frac{1}{A_{4}}-\frac{\left(\alpha_{3}+\alpha_{4}\right)^{2} m_{N}^{2}-\alpha_{3} \alpha_{4} q^{2}}{A_{4}^{2}}\right] \gamma_{\mu}\right. \\
& \left.+\left[\frac{\left(\alpha_{3}+\alpha_{4}\right)^{2} m_{N}}{A_{4}^{2}}-\frac{\left(\alpha_{3}+\alpha_{4}\right)}{A_{4}} \delta\right]\left(p^{\prime}+p\right)_{\mu}\right\}
\end{aligned}
$$

em que definimos

$$
f_{b}\left(\alpha_{i}, q^{2}\right)=\alpha_{1} \Lambda_{\kappa}^{2}+\alpha_{2} m_{\kappa}^{2}+\left(\alpha_{3}+\alpha_{4}\right) \delta_{2}+\frac{\left(\alpha_{3}+\alpha_{4}\right)^{2} m_{N}^{2}-\alpha_{3} \alpha_{4} q^{2}}{A_{4}}
$$

e também a função do cut-off $c\left(\Lambda_{\kappa}^{2}\right)$, que vai aparecer na resolução das integrais das outras amplitudes,

$$
c\left(\Lambda_{\kappa}^{2}\right)=\frac{g_{N \kappa Y}^{2}}{(4 \pi)^{2}}\left(m_{\kappa}^{2}-\Lambda_{\kappa}^{2}\right)^{2}
$$


Depois de separar os termos proporcionais a $\left(p^{\prime}+p\right)$, vamos usar a identidade de Gordon para fazer a substituição $\left(p^{\prime}+p\right)_{\mu} \rightarrow 2 m_{N} \gamma_{\mu}-i \sigma_{\mu \nu} q^{\nu}$. Note que é a identidade de Gordon que introduz os termos proporcionais a $\sigma_{\mu \nu}$ e que nos permite, posteriormente, identificar os fatores de forma de Dirac e Pauli de acordo com a Eq. (3.2).

Após usar a identidade de Gordon, escrevemos a expressão final para a amplitude de Feynman para o acoplamento bariônico,

$$
\begin{aligned}
\Gamma_{\mu}^{Y}\left(q^{2}\right)= & c\left(\Lambda_{\kappa}^{2}\right) Q_{Y} \int_{0}^{\infty} \prod_{i=1}^{4} d \alpha_{i} \frac{\alpha_{1}}{A_{4}^{2}} \exp \left(-f_{b}\left(\alpha_{i}, q^{2}\right)\right) \\
& \left\{\left[\delta^{2}+\frac{1-2\left(\alpha_{3}+\alpha_{4}\right) m_{N} \delta}{A_{4}}+\frac{\left(\alpha_{3}+\alpha_{4}\right)^{2} m_{N}^{2}+\alpha_{3} \alpha_{4} q^{2}}{A_{4}^{2}}\right] \gamma_{\mu}\right. \\
& \left.+2 m_{N}\left[\frac{\left(\alpha_{3}+\alpha_{4}\right) \delta}{A_{4}}-\frac{\left(\alpha_{3}+\alpha_{4}\right)^{2} m_{N}}{A_{4}^{2}}\right] \frac{i \sigma_{\mu \nu}}{2 m_{N}} q^{\nu}\right\}
\end{aligned}
$$

e isolamos a contribuição da amplitude $\Gamma_{\mu}^{Y}$ para os fatores de forma de Pauli e Dirac,

$$
\begin{aligned}
F_{1 Y}^{s}\left(q^{2}\right)= & c\left(\Lambda_{\kappa}^{2}\right) Q_{Y} \int_{0}^{\infty} \prod_{i=1}^{4} d \alpha_{i} \frac{\alpha_{1}}{A_{4}^{2}} \exp \left(-f_{b}\left(\alpha_{i}, q^{2}\right)\right) \\
& {\left[\delta^{2}+\frac{1-2 m_{N} \delta\left(\alpha_{3}+\alpha_{4}\right)}{A_{4}}+\frac{\left(\alpha_{3}+\alpha_{4}\right)^{2} m_{N}^{2}+\alpha_{3} \alpha_{4} q^{2}}{A_{4}^{2}}\right], } \\
F_{2 Y}^{s}\left(q^{2}\right)= & 2 m_{N} c\left(\Lambda_{\kappa}^{2}\right) Q_{Y} \int_{0}^{\infty} \prod_{i=1}^{4} d \alpha_{i} \frac{\alpha_{1}\left(\alpha_{3}+\alpha_{4}\right)}{A_{4}^{3}} \exp \left(-f_{b}\left(\alpha_{i}, q^{2}\right)\right) \\
& {\left[\delta-\frac{\left(\alpha_{3}+\alpha_{4}\right) m_{N}}{A_{4}}\right] . }
\end{aligned}
$$

Os integrandos em (4.17) e (4.18) são compostos pela multiplicação de dois termos que dependem não linearmente das variáveis $\alpha_{i}$. Para facilitar o cálculo, vamos separar a integração da exponencial, introduzindo a integral

$$
\int_{0}^{\infty} d \lambda \delta\left(\lambda-A_{4}\right)=1
$$

e a escala $\alpha_{i} \rightarrow \lambda \alpha_{i}$ nas expressões dos fatores de forma. Note que a inclusão da escala implica que $f_{b}\left(\alpha_{i}, q^{2}\right) \rightarrow \lambda f_{b}\left(\alpha_{i}, q^{2}\right)$ e que

$$
\int_{0}^{\infty} d \lambda \delta\left(\lambda-A_{4}\right)=\int_{0}^{\infty} \frac{d \lambda}{\lambda} \delta\left(1-A_{4}\right)
$$

de modo que reescrevemos as expressões de $F_{1 Y}^{s}$ e $F_{2 Y}^{s}$ como 


$$
\begin{aligned}
F_{1 Y}^{s}\left(q^{2}\right)= & c\left(\Lambda_{\kappa}^{2}\right) Q_{Y} \int_{0}^{\infty} \prod_{i=1}^{4} d \alpha_{i} \alpha_{1} \delta\left(1-A_{4}\right) \int_{0}^{\infty} d \lambda \lambda \exp \left(-\lambda f_{b}\left(\alpha_{i}, q^{2}\right)\right) \\
& \left\{1+\lambda\left[\delta^{2}-2\left(\alpha_{3}+\alpha_{4}\right) m_{N}+\left(\alpha_{3}+\alpha_{4}\right)^{2} m_{N}^{2}+\alpha_{3} \alpha_{4} q^{2}\right]\right\}, \\
F_{2 Y}^{s}\left(q^{2}\right)= & 2 m_{N} c\left(\Lambda_{\kappa}^{2}\right) Q_{Y} \int_{0}^{\infty} \prod_{i=1}^{4} d \alpha_{i} \alpha_{1}\left(\alpha_{3}+\alpha_{4}\right) \delta\left(1-A_{4}\right) \\
& {\left[\delta-\left(\alpha_{3}+\alpha_{4}\right) m_{N}\right] \int_{0}^{\infty} d \lambda \lambda^{2} \exp \left(-\lambda f_{b}\left(\alpha_{i}, q^{2}\right)\right) . }
\end{aligned}
$$

Integrando na variável $\lambda$ e usando a delta de Dirac para integrar em $\alpha_{4}$, obtemos finalmente,

$$
\begin{aligned}
& F_{1 Y}^{s}\left(q^{2}\right)=c\left(\Lambda_{\kappa}^{2}\right) Q_{Y} \int_{0}^{1} \prod_{i=1}^{3} d \alpha_{i} \theta\left(\alpha_{4}\right) \frac{\alpha_{1}}{f_{b}^{2}\left(\alpha_{i}, q^{2}\right)}\left[1+2 \frac{g_{b}^{2}\left(\alpha_{i}, q^{2}\right)}{f_{b}^{2}\left(\alpha_{i}, q^{2}\right)}\right], \\
& F_{2 Y}^{s}\left(q^{2}\right)=2 m_{N} c\left(\Lambda_{\kappa}^{2}\right) Q_{Y} \int_{0}^{1} \prod_{i=1}^{3} d \alpha_{i} \theta\left(\alpha_{4}\right) \frac{\alpha_{1}\left(\alpha_{3}+\alpha_{4}\right)}{f_{b}^{3}\left(\alpha_{i}, q^{2}\right)}\left[\delta-\left(\alpha_{3}+\alpha_{4}\right) m_{N}\right],
\end{aligned}
$$

nas quais temos $\alpha_{4}=1-\sum_{i=1}^{3} \alpha_{i}$ e as funções

$$
\begin{aligned}
& f_{b}\left(\alpha_{i}, q^{2}\right)=\alpha_{1} \Lambda_{\kappa}^{2}+\alpha_{2} m_{\kappa}^{2}+\left(\alpha_{3}+\alpha_{4}\right) \delta_{2}+\left(\alpha_{3}+\alpha_{4}\right)^{2} m_{N}^{2}-\alpha_{3} \alpha_{4} q^{2}, \\
& g_{b}\left(\alpha_{i}, q^{2}\right)=\delta^{2}-2\left(\alpha_{3}+\alpha_{4}\right) m_{N} \delta+\left(\alpha_{3}+\alpha_{4}\right)^{2} m_{N}^{2}+\alpha_{3} \alpha_{4} q^{2} .
\end{aligned}
$$

\subsection{Acoplamento mesônico}

Partindo da Eq. (3.60), temos para o acoplamento da corrente com o méson:

$$
\begin{aligned}
\Gamma_{\mu}^{\kappa}\left(p, p^{\prime}\right) & =i g_{N \kappa Y}^{2} Q_{\kappa}\left(m_{\kappa}^{2}-\Lambda_{\kappa}^{2}\right)^{2} \int \frac{d^{4} k}{(2 \pi)^{4}}(2 k+q)_{\mu}\left(\not p-\not k+m_{Y}\right) \\
& \times \frac{1}{\left(k^{2}-\Lambda_{\kappa}^{2}\right)\left(k^{2}-m_{\kappa}^{2}\right)\left[(p-k)^{2}-m_{Y}^{2}\right]\left[(k+q)^{2}-m_{\kappa}^{2}\right]\left[(k+q)^{2}-\Lambda_{\kappa}^{2}\right]}
\end{aligned}
$$

Depois de usar a parametrização de Schwinger e a equação de Dirac para reescrever a equação anterior, integramos na variável $k$. Com isso, 


$$
\begin{aligned}
\Gamma_{\mu}^{\kappa}\left(p, p^{\prime}\right)= & c\left(\Lambda_{\kappa}^{2}\right) Q_{\kappa} \int_{0}^{\infty} \prod_{i=1}^{5} d \alpha_{i} \frac{1}{A_{5}^{3}} \exp \left(-f_{m}\left(\alpha_{i}, q^{2}\right)\right) \\
& \times\left\{\left(\delta-\frac{\alpha_{3} m_{N}}{A_{5}}\right)\left[\alpha_{3}\left(p^{\prime}+p\right)_{\mu}-\left(\left(\alpha_{1}-\alpha_{4}\right)+\left(\alpha_{2}-\alpha_{5}\right)\right) q_{\mu}\right]+\gamma_{\mu}\right\}
\end{aligned}
$$

depois de considerar que entre espinores de Dirac termos proporcionais a $\not q=\not p^{\prime}-\not p$ se anulam e $\not p \rightarrow m_{N}$, e usar $\left[\left(\alpha_{1}+\alpha_{2}\right) q-\alpha_{3} p\right]^{2}=\left(\alpha_{1}+\alpha_{2}\right)\left(\alpha_{1}+\alpha_{2}+\alpha_{3}\right) q^{2}+\alpha_{3}^{2} m_{N}^{2}$. Na Eq. (4.28), definimos $A_{5}=\sum_{i=1}^{5} \alpha_{i}$ e

$$
\begin{aligned}
f_{m}\left(\alpha_{i}, q^{2}\right) & =\left(\alpha_{1}+\alpha_{4}\right) m_{\kappa}^{2}+\left(\alpha_{2}+\alpha_{5}\right) \Lambda_{\kappa}^{2}+\alpha_{3} \delta_{2} \\
& +\frac{\alpha_{3}^{2} m_{N}^{2}-\left(\alpha_{1}+\alpha_{2}\right)\left(\alpha_{4}+\alpha_{5}\right) q^{2}}{A_{5}} .
\end{aligned}
$$

Note que o integrando da Eq. (4.28) é simétrico pelas trocas $\alpha_{1} \leftrightarrow \alpha_{4}$ e $\alpha_{2} \leftrightarrow \alpha_{5}$, de forma que a integral dos termos proporcionais a $\left(\alpha_{1}-\alpha_{4}\right)$ e $\left(\alpha_{2}-\alpha_{5}\right)$ é nula. Usando isso e a identidade de Gordon, a expressão final da amplitude para o acoplamento mesônico é dada por

$$
\begin{aligned}
\Gamma_{\mu}^{\kappa}\left(p, p^{\prime}\right)= & c\left(\Lambda_{\kappa}^{2}\right) Q_{\kappa} \int_{0}^{\infty} \prod_{i=1}^{5} d \alpha_{i} \frac{1}{A_{5}^{3}} \exp \left(-f_{m}\left(\alpha_{i}, q^{2}\right)\right) \\
& \times\left\{\left[1+2 \alpha_{3} m_{N}\left(\delta-\frac{\alpha_{3} m_{N}}{A_{5}}\right)\right] \gamma_{\mu}-2 \alpha_{3} m_{N}\left(\delta-\frac{\alpha_{3} m_{N}}{A_{5}}\right) \frac{i \sigma_{\mu \nu}}{2 m_{N}} q^{\nu}\right\},
\end{aligned}
$$

da qual identificamos as contribuições de $\Gamma_{\mu}^{\kappa}$ para os fatores de forma,

$$
\begin{aligned}
F_{1 \kappa}^{s}\left(q^{2}\right)= & c\left(\Lambda_{\kappa}^{2}\right) Q_{\kappa} \int_{0}^{\infty} \prod_{i=1}^{5} d \alpha_{i} \frac{1}{A_{5}^{3}} \exp \left(-f_{m}\left(\alpha_{i}, q^{2}\right)\right) \\
& \times\left[1+2 \alpha_{3} m_{N}\left(\delta-\frac{\alpha_{3} m_{N}}{A_{5}}\right)\right], \\
F_{2 \kappa}^{s}\left(q^{2}\right)= & -2 m_{N} c\left(\Lambda_{\kappa}^{2}\right) Q_{\kappa} \int_{0}^{\infty} \prod_{i=1}^{5} d \alpha_{i} \frac{\alpha_{3}}{A_{5}^{3}} \exp \left(-f_{m}\left(\alpha_{i}, q^{2}\right)\right) \\
& \times\left(\delta-\frac{\alpha_{3} m_{N}}{A_{5}}\right) .
\end{aligned}
$$


Aplicando o mesmo método usado na seção anterior para integrar a exponencial, reescrevemos os fatores de forma como

$$
\begin{aligned}
& F_{1 \kappa}^{s}\left(q^{2}\right)=c\left(\Lambda_{\kappa}^{2}\right) Q_{\kappa} \int_{0}^{1} \prod_{i=1}^{4} d \alpha_{i} \theta\left(\alpha_{5}\right) \frac{1}{f_{m}^{2}\left(\alpha_{i}, q^{2}\right)}\left[1+4 \frac{\alpha_{3} m_{N}\left(\delta-\alpha_{3} m_{N}\right)}{f_{m}\left(\alpha_{i}, q^{2}\right)}\right], \\
& F_{2 \kappa}^{s}\left(q^{2}\right)=-4 m_{N} c\left(\Lambda_{\kappa}^{2}\right) Q_{\kappa} \int_{0}^{1} \prod_{i=1}^{4} d \alpha_{i} \theta\left(\alpha_{5}\right) \frac{\alpha_{3}}{f_{m}^{3}\left(\alpha_{i}, q^{2}\right)}\left(\delta-\alpha_{3} m_{N}\right),
\end{aligned}
$$

em que $\alpha_{5}=1-\sum_{i=1}^{4} \alpha_{i}$ e

$$
\begin{aligned}
f_{m}\left(\alpha_{i}, q^{2}\right) & =\left(\alpha_{1}+\alpha_{4}\right) m_{\kappa}^{2}+\left(\alpha_{2}+\alpha_{5}\right) \Lambda_{\kappa}^{2}+\alpha_{3} \delta_{2}+\alpha_{3}^{2} m_{N}^{2} \\
& -\left(\alpha_{1}+\alpha_{2}\right)\left(\alpha_{4}+\alpha_{5}\right) q^{2}
\end{aligned}
$$

\subsection{Acoplamento com o vértice não pontual}

No caso do acoplamento com o vértice, reescrevemos as amplitudes dadas nas Eqs. (3.61) e (3.62) como

$$
\begin{aligned}
\Gamma_{\mu}^{v 1}\left(p, p^{\prime}\right)= & -i g_{N \kappa Y}^{2} Q_{\kappa}\left(m_{\kappa}^{2}-\Lambda_{\kappa}^{2}\right)^{2} \int \frac{d^{4} k}{(2 \pi)^{4}}(q-2 k)_{\mu}\left(\not p^{\prime}-\not k+m_{Y}\right) \\
& \frac{1}{\left(k^{2}-\Lambda_{\kappa}^{2}\right)^{2}\left(k^{2}-m_{\kappa}^{2}\right)\left[(k-q)^{2}-\Lambda_{\kappa}^{2}\right]\left[\left(p^{\prime}-k\right)^{2}-m_{Y}^{2}\right]}, \\
\Gamma_{\mu}^{v 2}\left(p, p^{\prime}\right)= & i g_{N \kappa Y}^{2} Q_{\kappa}\left(m_{\kappa}^{2}-\Lambda_{\kappa}^{2}\right)^{2} \int \frac{d^{4} k}{(2 \pi)^{4}}(q+2 k)_{\mu}\left(\not p-\not k+m_{Y}\right) \\
& \frac{1}{\left(k^{2}-\Lambda_{\kappa}^{2}\right)^{2}\left(k^{2}-m_{\kappa}^{2}\right)\left[(k+q)^{2}-\Lambda_{\kappa}^{2}\right]\left[(p-k)^{2}-m_{Y}^{2}\right]} .
\end{aligned}
$$

Usando a equação de Dirac, a fórmula de Schwinger e integrando na variável $k$, temos

$$
\begin{aligned}
\Gamma_{\mu}^{v 1}\left(p, p^{\prime}\right)= & -c\left(\Lambda_{\kappa}^{2}\right) Q_{\kappa} \int_{0}^{\infty} \prod_{i=1}^{4} d \alpha_{i} \frac{\alpha_{1}}{A_{4}^{2}} \exp \left(-f_{v}\left(\alpha_{i}, q^{2}\right)\right) \\
& \times\left[\delta q_{\mu}-\frac{\gamma_{\mu}+\alpha_{3} m_{N} q_{\mu}+2 \delta\left(\alpha_{3} p^{\prime}+\alpha_{4} q\right)_{\mu}}{A_{4}}+\frac{2 \alpha_{3}\left(\alpha_{3} p^{\prime}+\alpha_{4} q\right)_{\mu} m_{N}}{A_{4}^{2}}\right],
\end{aligned}
$$




$$
\begin{aligned}
\Gamma_{\mu}^{v 2}\left(p, p^{\prime}\right)= & -c\left(\Lambda_{\kappa}^{2}\right) Q_{\kappa} \int_{0}^{\infty} \prod_{i=1}^{4} d \alpha_{i} \frac{\alpha_{1}}{A_{4}^{2}} \exp \left(-f_{v}\left(\alpha_{i}, q^{2}\right)\right) \\
& \times\left[-\delta q_{\mu}-\frac{\gamma_{\mu}-\alpha_{3} m_{N} q_{\mu}+2 \delta\left(\alpha_{3} p-\alpha_{4} q\right)_{\mu}}{A_{4}}+\frac{2 \alpha_{3}\left(\alpha_{3} p-\alpha_{4} q\right)_{\mu} m_{N}}{A_{4}^{2}}\right],
\end{aligned}
$$

em que consideramos que entre espinores de Dirac os termos proporcionais a $\not q=\not p^{\prime}-\not p$ se anulam e $\not p^{\prime} \rightarrow m_{N}$, e definimos

$$
\begin{aligned}
f_{v}\left(\alpha_{i}, q^{2}\right) & =\left(\alpha_{1}+\alpha_{4}\right) \Lambda_{\kappa}^{2}+\alpha_{2} m_{\kappa}+\alpha_{3} \delta_{2} \\
& +\frac{\alpha_{3}^{2} m_{N}^{2}-\alpha_{4}\left(\alpha_{1}+\alpha_{2}\right) q^{2}}{A_{4}} .
\end{aligned}
$$

Somando as Eqs. (4.38) e (4.39) e substituindo a identidade de Gordon, escrevemos a expressão final para a amplitude do acoplamento com o vértice,

$$
\begin{aligned}
\Gamma_{\mu}^{v}\left(p, p^{\prime}\right)= & -c\left(\Lambda_{\kappa}^{2}\right) Q_{\kappa} \int_{0}^{\infty} \prod_{i=1}^{4} d \alpha_{i} \frac{1}{A_{4}^{3}} \exp \left(-f_{v}\left(\alpha_{i}, q^{2}\right)\right) \\
& \times\left[\left(\frac{4 \alpha_{3}^{2} m_{N}^{2}}{A_{4}}-4 \alpha_{3} \delta m_{N}-2\right) \gamma_{\mu}-\left(\frac{4 \alpha_{3}^{2} m_{N}^{2}}{A_{4}}-4 \alpha_{3} \delta m_{N}\right) \frac{i \sigma_{\mu \nu}}{2 m_{N}} q^{\nu}\right]
\end{aligned}
$$

e identificamos as contribuições

$$
\begin{aligned}
F_{1 v}^{s}\left(q^{2}\right)= & -2 c\left(\Lambda_{\kappa}^{2}\right) Q_{\kappa} \int_{0}^{\infty} \prod_{i=1}^{4} d \alpha_{i} \frac{\alpha_{1}}{A_{4}^{3}} \exp \left(-f_{v}\left(\alpha_{i}, q^{2}\right)\right) \\
& {\left[\frac{2 \alpha_{3}^{2} m_{N}^{2}}{A_{4}}-2 \alpha_{3} \delta m_{N}-1\right], } \\
F_{2 v}^{s}\left(q^{2}\right)= & -4 m_{N} c\left(\Lambda_{\kappa}^{2}\right) Q_{\kappa} \int_{0}^{\infty} \prod_{i=1}^{4} d \alpha_{i} \frac{\alpha_{1} \alpha_{3}}{A_{4}^{3}} \exp \left(-f_{v}\left(\alpha_{i}, q^{2}\right)\right) \\
& {\left[\delta-\frac{\alpha_{3} m_{N}}{A_{4}}\right], }
\end{aligned}
$$

para os fatores de forma. Assim como nas expressões dos outros acoplamentos, vamos introduzir a escala e a delta de Dirac para integrar a exponencial. Por fim, integrando na variável $\alpha_{4}$, temos 


$$
\begin{aligned}
& F_{1 v}^{s}\left(q^{2}\right)=2 c\left(\Lambda_{\kappa}^{2}\right) Q_{\kappa} \int_{0}^{1} \prod_{i=1}^{3} d \alpha_{i} \theta\left(\alpha_{4}\right) \frac{\alpha_{1}}{f_{v}^{2}\left(\alpha_{i}, q^{2}\right)}\left[1-\frac{4 \alpha_{3} m_{N}\left(\alpha_{3} m_{N}-\delta\right)}{f_{v}\left(\alpha_{i}, q^{2}\right)}\right], \\
& F_{2 v}^{s}\left(q^{2}\right)=8 m_{N} c\left(\Lambda_{\kappa}^{2}\right) Q_{\kappa} \int_{0}^{1} \prod_{i=1}^{3} d \alpha_{i} \theta\left(\alpha_{4}\right) \frac{\alpha_{1} \alpha_{3}}{f_{v}^{3}\left(\alpha_{i}, q^{2}\right)}\left(\alpha_{3} m_{N}-\delta\right),
\end{aligned}
$$

nas quais $\alpha_{4}=1-\sum_{i=1}^{3} \alpha_{i} \mathrm{e}$

$$
f_{v}\left(\alpha_{i}, q^{2}\right)=\left(\alpha_{1}+\alpha_{4}\right) \Lambda_{\kappa}^{2}+\alpha_{2} m_{\kappa}+\alpha_{3} \delta_{2}+\left(\alpha_{3}^{2} m_{N}^{2}-\alpha_{4}\right)\left(\alpha_{1}+\alpha_{2}\right) q^{2} .
$$




\section{Capítulo 5}

\section{Resultados numéricos}

Dispondo de todas as contribuições calculadas nas seções anteriores, determinamos os fatores de forma estranhos de Pauli e Dirac,

$$
\begin{aligned}
& F_{1}^{s}\left(q^{2}\right)=\sum_{Y=\Lambda, \Sigma}\left[F_{1 Y}^{s}\left(q^{2}\right)+F_{1 \kappa}^{s}\left(q^{2}\right)+F_{1 v}^{s}\left(q^{2}\right)\right] \\
& F_{2}^{s}\left(q^{2}\right)=\sum_{Y=\Lambda, \Sigma}\left[F_{2 Y}^{s}\left(q^{2}\right)+F_{2 \kappa}^{s}\left(q^{2}\right)+F_{2 v}^{s}\left(q^{2}\right)\right],
\end{aligned}
$$

respectivamente. Em seguida calculamos $G_{E}^{s}$ e $G_{M}^{s}$, dados em função de $F_{1}^{s}$ e $F_{2}^{s}$ pelas Eqs. (2.48) e (2.49), e também a combinação (2.65), que é obtida experimentalmente e por isso deve ser explicada pelo nosso modelo.

Fizemos as contas analiticamente até chegar nas Eqs. (4.23) e (4.24) para $F_{1,2 Y}^{s}$, (4.33) e (4.34) para $F_{1,2 \kappa}^{s}$, e (4.44) e (4.45) para $F_{1,2 v}^{s}$, e a partir destas expressões continuamos os cálculos numericamente. Alguns resultados preliminares que incluiam apenas a contribuição do estado intermediário $|\kappa ; \Lambda\rangle$ já foram publicados em 2007 [38]. Após a publicação deste trabalho incluimos o estado $|\kappa ; \Sigma\rangle$ no cálculo, e os resultados obtidos para a soma dos dois estados são apresentados e discutidos neste capítulo.

\subsection{Constantes e parâmetros}

Os fatores de forma nas Eqs. (2.48) e (2.49) dependem das massas das partículas envolvidas e das constantes de acoplamento. Para as massas do próton e dos híperons, temos [29] 


$$
\begin{aligned}
& m_{N}=938 \mathrm{MeV}, \\
& m_{\Lambda}=1116 \mathrm{MeV}, \\
& m_{\Sigma}=1190 \mathrm{MeV} .
\end{aligned}
$$

e para a massa do méson $\kappa[22]$,

$$
m_{\kappa}=797 \pm 19 \pm 42 \mathrm{MeV}
$$

Para as constantes de acoplamento, usamos os valores do potencial de Nijmegen no modelo NSC97f [39],

$$
\begin{aligned}
& g_{N \kappa \Lambda} \approx-10,0, \\
& g_{N \kappa \Sigma} \approx-6,7 .
\end{aligned}
$$

A combinação (2.65) dos fatores de forma depende do parâmetro $\eta$, dado pela Eq. (2.64), que é uma função do momento trocado $q^{2}$ e dos fatores de forma eletromagnéticos do próton. Para determinar o valor de $\eta$ para cada $q^{2}$, usamos a parametrização de Kelly $\operatorname{para} G_{E}^{\gamma p}$ e $G_{M}^{\gamma p}[41]$.

Além das constantes e do parâmetro $\eta$, os resultados também dependem de parâmetros de cut-off que aparecem quando consideramos o vértice extenso, tanto no caso dos estados com o $\kappa$ calculados neste trabalho, quanto no caso de estados com o káon calculados anteriormente pelo grupo [21]. Na análise numérica nós variamos os cut-offs correspondentes ao káon e ao $\kappa$ dentro do intervalo $0,9 \mathrm{GeV} \leq \Lambda_{M} \leq 1,1 \mathrm{GeV}$, o que está bem próximo dos valores das Refs. [39] e [40]. Depois de alguns cálculos foi notado que a contribuição do $\kappa$ tende a cancelar a contribuição do káon em todos os observáveis considerados. Uma vez que, de acordo com a Eq. (4.15), o aumento do cut-off leva diretamente ao aumento da contribuição do méson correspondente, existem duas escolhas extremas: I) $\Lambda_{\kappa}=0,9 \mathrm{GeV}$ e $\Lambda_{K}=1,1 \mathrm{GeV}$, que maximiza a contribuição do káon, e II) $\Lambda_{\kappa}=1,1 \mathrm{GeV}$ e $\Lambda_{K}=0,9 \mathrm{GeV}$, que maximiza a contribuição do $\kappa$. Nas seções a seguir mostraremos os resultados para cada uma destas escolhas.

Os fatores de forma estranhos e a combinação deles são funções de $q^{2}$, mas eles foram calculados e depois graficados em função da variável $Q^{2}=-q^{2} \geq 0$. Essa mudança de variável é conveniente porque o momento trocado no espalhamento elástico ep é tipo espaço. Variamos o momento trocado dentro do intervalo $0 \leq Q^{2} \leq 1,2 \mathrm{GeV}^{2}$ de modo a cobrir toda a gama de dados disponíveis atualmente. 


\subsection{Fator de forma elétrico estranho}

No gráfico da Fig. 5.1 comparamos os dados disponíveis para $G_{E}^{s}$ com os resultados do nosso modelo, obtidos com as escolhas I (linha azul) e II (linha vermelha) para os cut-offs. Veja que, quantitativamente, o modelo está de acordo com os dados das colaborações A4 e HAPPEX e da análise global feita por Pate, e que não há uma alteração significativa neste resultado quando variamos os cut-offs dentro do intervalo pré-estabelecido. Além disso, é importante notar que $G_{E}^{s}(0)$ é compatível com zero nas duas curvas, o que está de acordo com o esperado pois não existe estranheza líquida no próton. Embora exista uma clara tendência dos dados que não é descrita pelas curvas teóricas, isto não é o suficiente para invalidar o nosso modelo, dada a incerteza dos pontos experimentais.

A influência de cada méson nos resultados da Fig. 5.1 é evidenciada na Fig. 5.2, em que a contribuição do káon (linha tracejada) e do $\kappa$ (linha cheia) são mostradas separadamente, para as escolhas I e II. Devido à ordem de grandeza da diferença entre as duas contribuições, principalmente na combinação I dos cut-offs, não é possível comparar a influência do káon com a do $\kappa$ e, ao mesmo tempo, equiparar estas curvas com os dados.

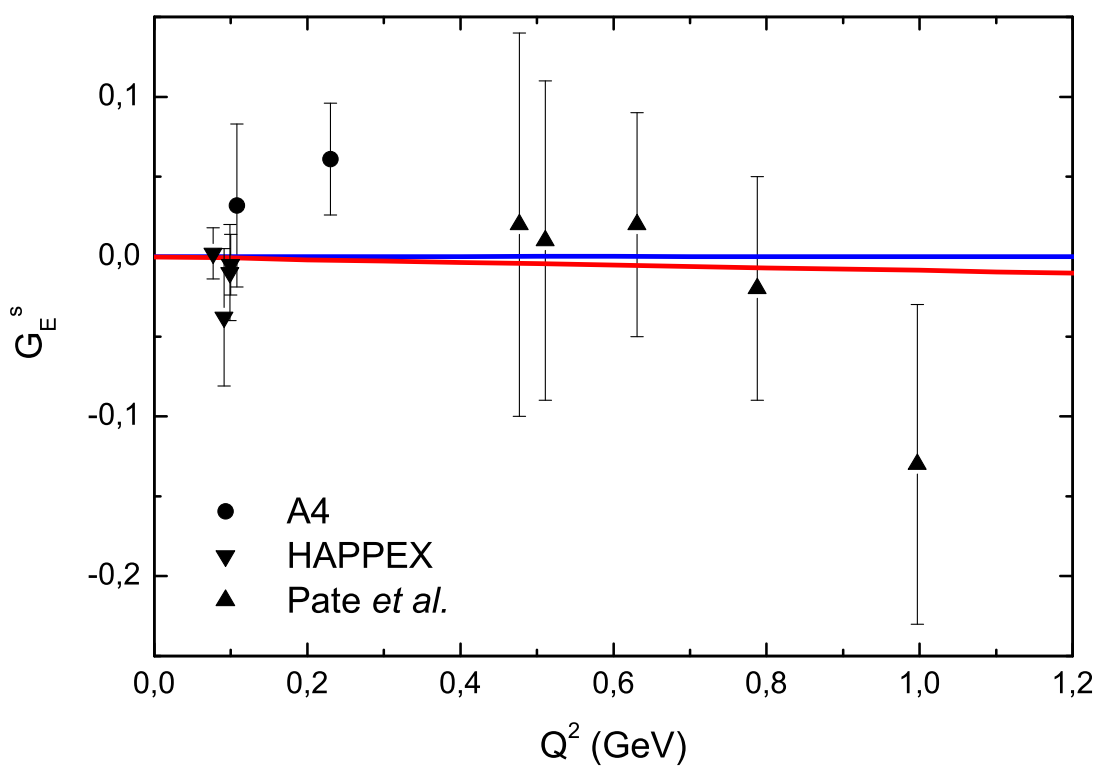

Figura 5.1: Fator de forma elétrico estranho. As linhas azul e vermelha mostram nossos resultados com $\Lambda_{\kappa}=0,9 \mathrm{GeV}, \Lambda_{K}=1,1 \mathrm{GeV}$ e $\Lambda_{\kappa}=1,1 \mathrm{GeV}, \Lambda_{K}=0,9 \mathrm{GeV}$, respectivamente. 


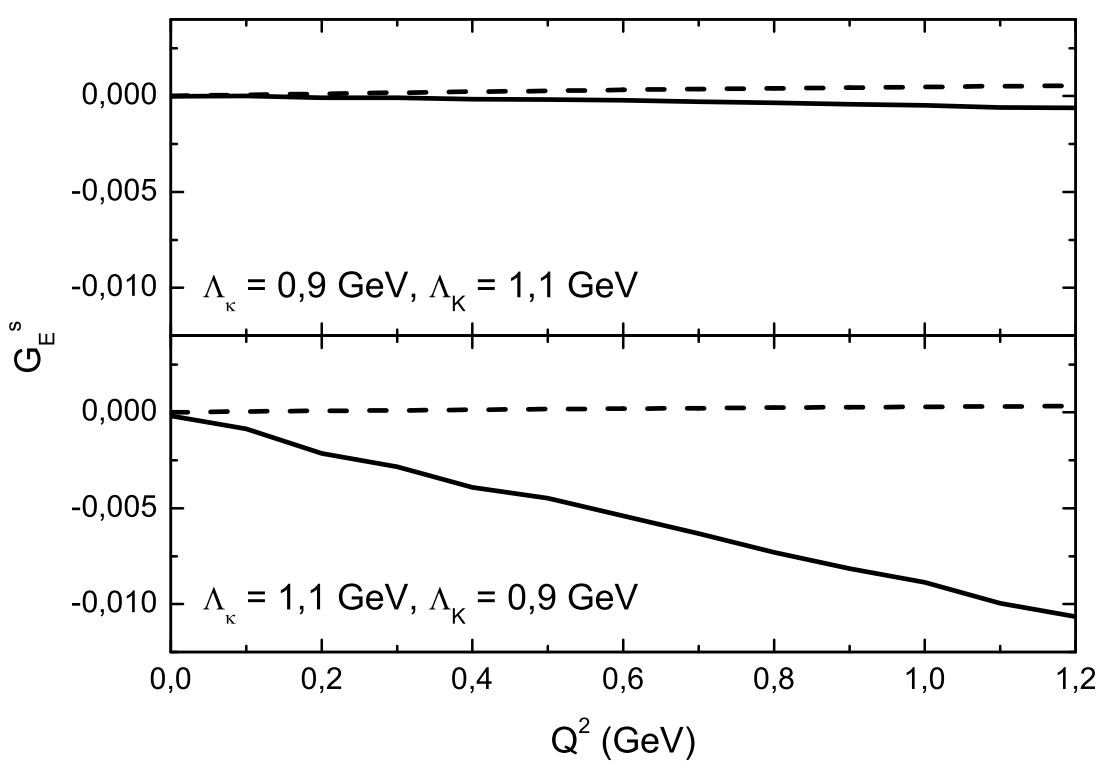

Figura 5.2: Contribuição do káon (linha tracejada) e do méson $\kappa$ (linha cheia) para o fator de forma elétrico estranho, calculada nas combinações de cut-offs I (acima) e II (abaixo).

Nos resultados da Fig. 5.2 vemos que a contribuição do káon para $G_{E}^{s}$, mesmo quando escolhemos os cut-offs que maximizam a sua contribuição, é praticamente zero, e por isso a contribuição do $\kappa$ é o que de fato define as curvas teóricas. Isto acontece porque as constantes de acoplamento do potencial de Nijmegen para vértices envolvendo o káon $\left(g_{N K \Lambda} \approx-1,2\right.$ e $\left.g_{N K \Sigma} \approx 0,4\right)$ são muito menores do que os valores para o $\kappa$, dados em (5.7) e (5.8). Lembrando que nas integrais de loop as constantes aparecem ao quadrado, podemos estimar a contribuição do káon com relação à contribuição do $\kappa$ :

$$
\begin{aligned}
& \left(\frac{g_{N K \Lambda}}{g_{N \kappa \Lambda}}\right)^{2} \approx 1,4 \% \\
& \left(\frac{g_{N K \Sigma}}{g_{N \kappa \Sigma}}\right)^{2} \approx 0,3 \%
\end{aligned}
$$

o que explica porque a contribuição do káon é bem menor que a do $\kappa$. 


\subsection{Fator de forma magnético estranho}

Uma análise idêntica à anterior também foi feita para $G_{M}^{s}$. Primeiro, comparamos os dados das colaborações HAPPEX e SAMPLE e da análise de Pate com os nossos resultados, calculados com as combinações I (linha azul) e II (linha vermelha) de cut-offs. Em seguida, separamos a contribuição do káon (linha pontilhada) e do $\kappa$ (linha cheia) para cada um dos dois casos. Estes resultados estão apresentados nos gráficos das Fig. 5.3 e 5.4, respectivamente. Aqui, novamente, temos um bom acordo com os dados experimentais e com os dados do Pate, para qualquer escolha de cut-offs dentro do intervalo considerado.

De acordo com a normalização dos fatores de forma, $G_{M}^{s}(0)$ deve ser igual à contribuição da estranheza para o momento magnético do próton, $\mu_{s}$. Ainda não há um consenso a respeito do valor desta grandeza, e nem ao menos sabemos se ela é positiva ou negativa. Em QCD na rede, por exemplo, os resultados encontrados variam entre -0,28 \pm 0,10 até $+0,05 \pm 0,06$ em unidades de magneton nuclear [42]. Em um artigo de revisão publicado por Beck e McKeown em 2001 [43] encontra-se um histórico dos valores de $\mu_{s}$ calculados em diversos modelos, e os resultados variam em um intervalo ainda maior, de $-0,75 \pm 30$ m.n. a +0,42 m.n. No presente modelo, vemos no gráfico da Fig. 5.3 que $\mu_{s}$ tem sinal positivo e está entre $0,01<\mu_{s}<0,1$ m.n.

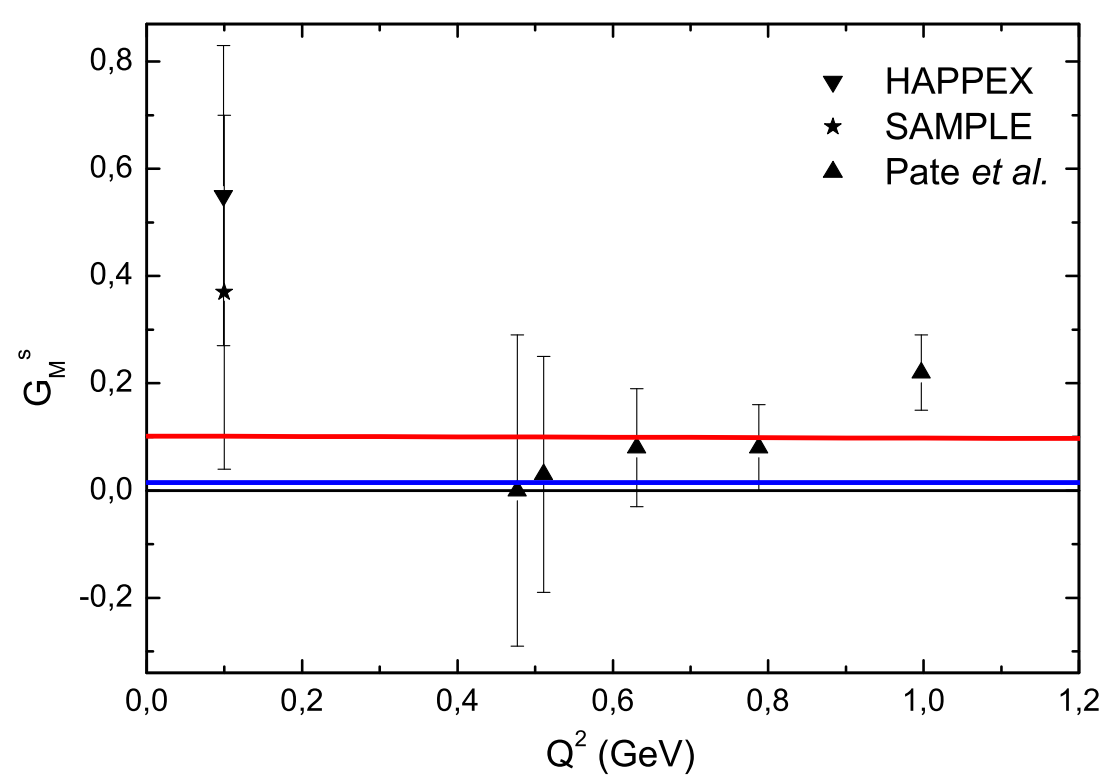

Figura 5.3: Idem à Fig. 5.1, mas para o fator de forma magnético estranho. 


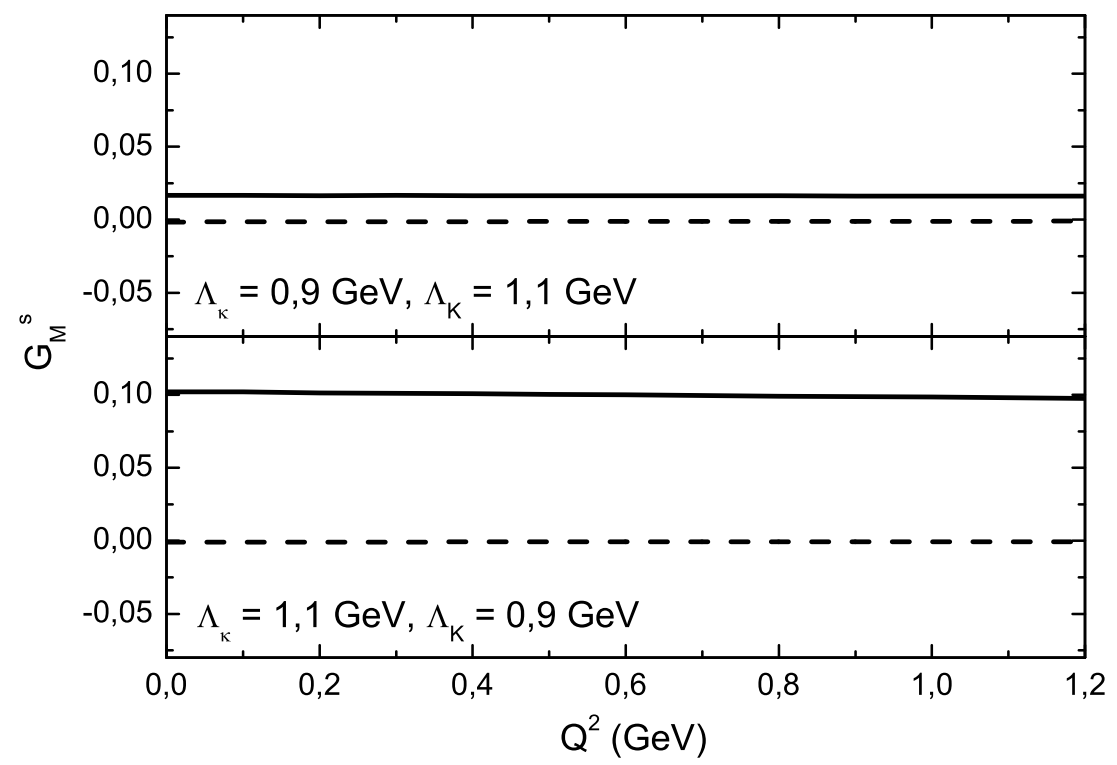

Figura 5.4: Idem à Fig. 5.2, mas para o fator de forma magnético estranho.

Nos gráficos da Fig. 5.4 vemos que a contribuição do káon é praticamente nula, até mesmo com a escolha de cut-offs que maximizam a sua importância, e que $G_{M}^{s}$ assume valores diferentes de zero somente por causa da inclusão do $\kappa$. Obviamente, as razões para isto são as mesmas discutidas no caso de $G_{E}^{s}$.

\subsection{Combinação $G_{E}^{s}+\eta G_{M}^{s}$}

Finalmente, apresentamos nesta seção os nossos resultados para a combinação $G_{E}^{s}+\eta G_{M}^{s}$ dos fatores de forma. Na Fig. 5.5 mostramos os resultados do modelo em comparação com os dados experimentais das colaborações A4, G0 e HAPPEX, para as duas escolhas extremas dos cut-offs. A contribuição do káon e do $\kappa$ são mostradas separadamente para a escolha I (Fig. 5.6) e II (Fig. 5.7).

De acordo com a Fig. 5.5, o modelo explica muito bem os dados para qualquer que seja a combinação de cut-offs entre as escolhas extremas I e II, mas quando maximizamos a contribuição do $\kappa$ a concordância é melhor e a curva teórica passa por mais pontos experimentais. Nas Figs. 5.6 e 5.7, vemos novamente que é a contribuição do $\kappa$ que faz com que a concordância entre modelo e dados seja satisfatória; a contribuição do káon, também neste caso, é praticamente zero. 


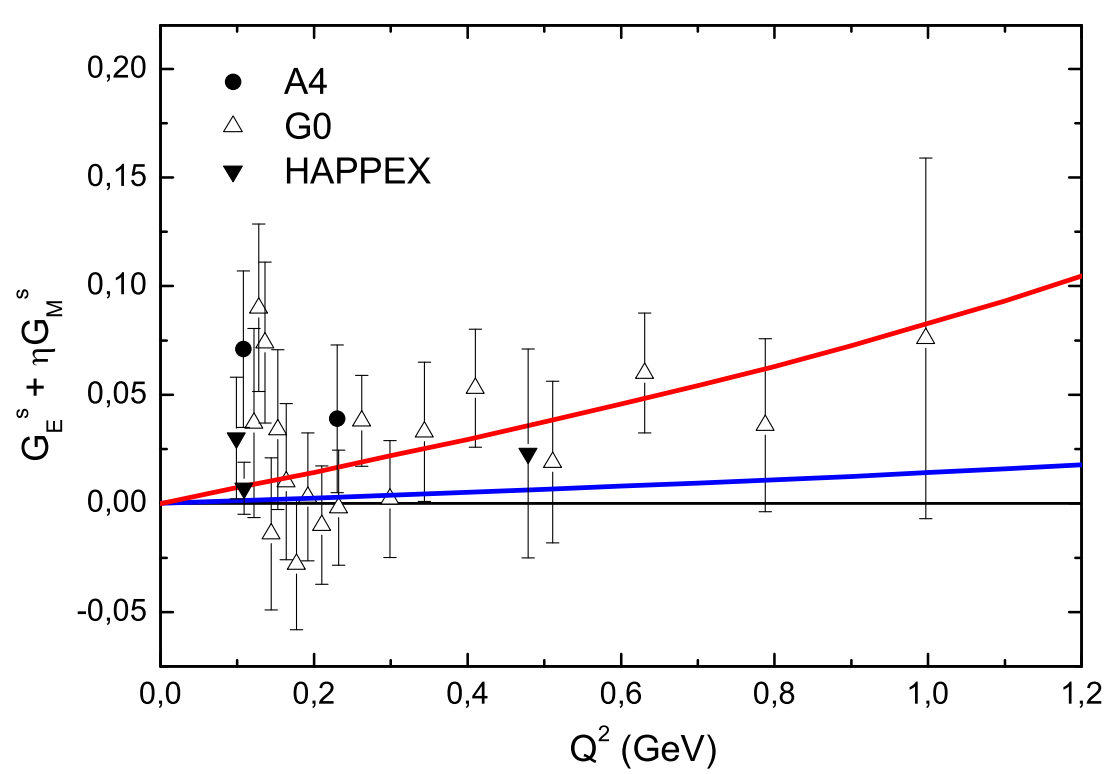

Figura 5.5: Idem à Fig. 5.1, mas para a combinação $G_{E}^{s}+\eta G_{M}^{s}$ dos fatores de forma estranhos.

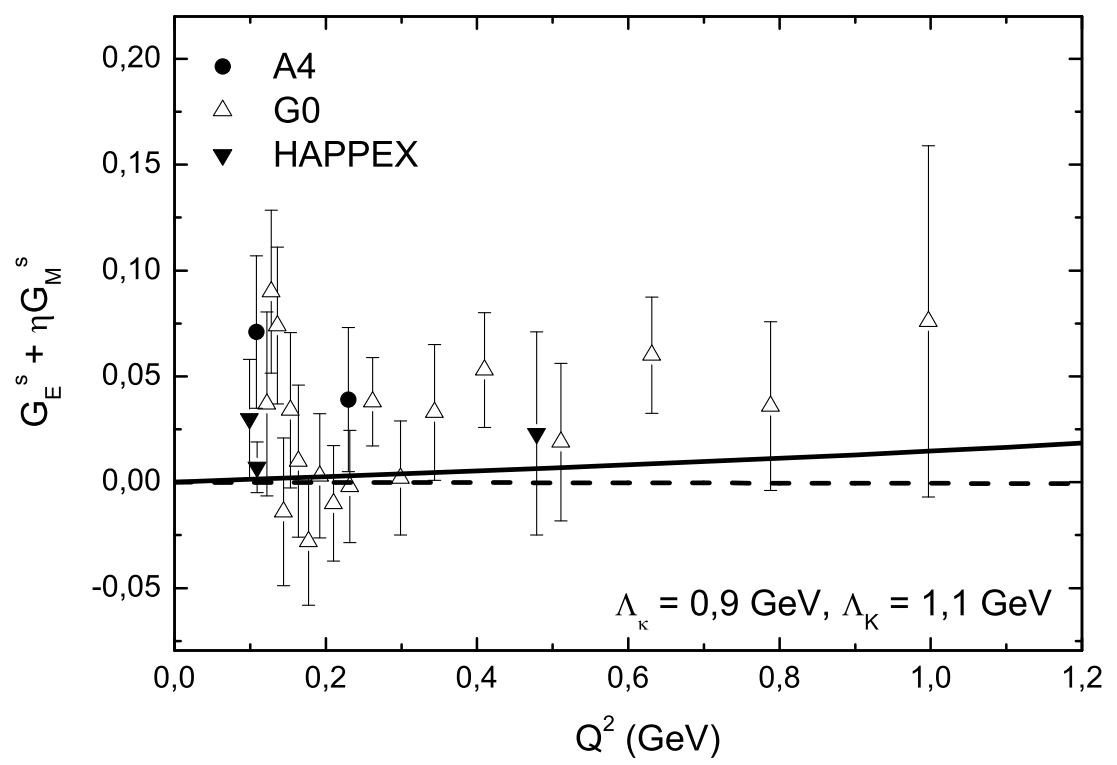

Figura 5.6: Contribuição do káon (linha tracejada) e do méson $\kappa$ (linha cheia) para o fator de forma elétrico estranho, na escolha I de cut-offs. 


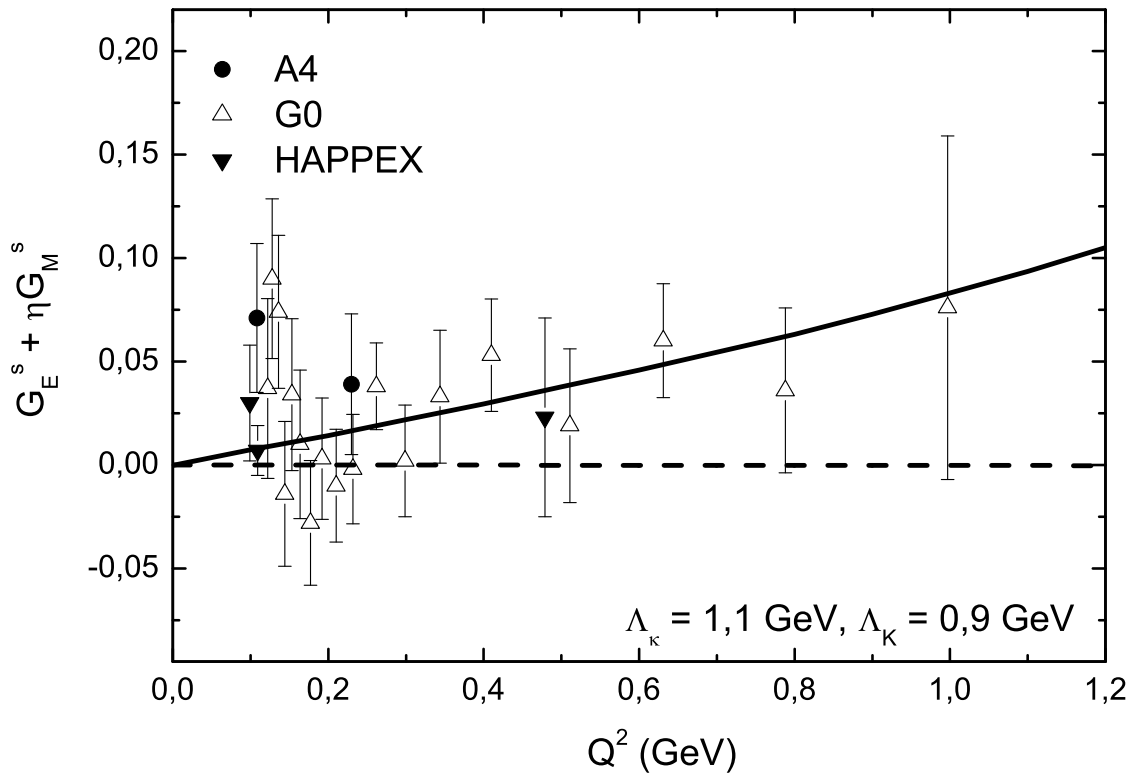

Figura 5.7: Idem à Fig. 5.6, mas na escolha II de cut-offs. 


\section{Capítulo 6}

\section{Conclusão}

Usamos uma versão do modelo da nuvem mesônica que inclui a contribuição do $\kappa$ para calcular $G_{E}^{s}$ e $G_{M}^{s}$ em função do momento trocado $Q^{2}$, dentro do intervalo $0 \leq Q^{2} \leq$ $1,2 \leq \mathrm{GeV}^{2}$, de modo a abranger todos os dados existentes no momento. Comparamos nossos resultados com os dados experimentais de $G_{E}^{s}, G_{M}^{s}$ e da combinação $G_{E}^{s}+\eta G_{M}^{s}$, bem como com os dados da análise global feita por Pate [23].

No caso de $G_{E}^{s}$, observamos um bom acordo quantitativo entre experimento e modelo para qualquer parâmetro de cut-off dentro do intervalo $0,9 \leq \Lambda \leq 1,1 \mathrm{GeV}$. Vimos que, conforme o esperado, $G_{E}^{s}(0)=0$ em todas as análises. Apesar de existir uma clara tendência dos dados que não pode ser explicada pelos nossos resultados, isto não invalida o modelo, dada a ordem de grandeza da incerteza nos dados.

Para $G_{M}^{s}$, verificamos que o modelo está de acordo com os dados, quantitativamente e qualitativamente, para qualquer escolha de cut-offs dentro do intervalo considerado. Os nossos valores de $G_{M}^{s}(0)$ indicam que contribuição da estranheza para o momento magnético do próton está dentro do intervalo $0,01<\mu_{s}<0,1$ m.n., e estes valores são próximos daqueles encontrados na literatura [42, 43].

A grande maioria dos dados experimentais são da combinação $G_{E}^{s}+\eta G_{M}^{s}$, para a qual obtivemos uma ótima concordância entre modelo e experimento. Os resultados são compatíveis com os dados para qualquer escolha de cut-offs do intervalo, mas a concordância é melhor quando usamos a combinação de cut-offs que maximiza a contribuição do $\kappa$.

Com estes resultados concluimos que o nosso modelo explica os dados existentes para os fatores de forma estranhos. Evidentemente, isto só foi possível devido à inclusão do 
$\kappa$ na nuvem, como podemos ver quando comparamos os nossos resultados com aqueles de um trabalho anterior do grupo [21], no qual se usou uma versão da nuvem incluindo somente com os mésons $K$ e $K^{*}$. Acreditamos que, neste sentido, o presente trabalho ajuda a corroborar ainda mais a existência deste méson escalar. 


\section{Apêndice A}

\section{Demonstração da Eq. (3.18)}

Deixamos para este apêndice a demonstração da Eq. (3.18), que usamos para provar a validade da identidade de Ward-Takahashi no caso do acoplamento bariônico. Esta equação é:

$$
S\left(p^{\prime}-k\right) \not q S(p-k)=i\left(S(p-k)-S\left(p^{\prime}-k\right)\right) .
$$

Comecemos calculando o primeiro termo da equação acima. Vamos definir

$$
I=S\left(p^{\prime}-k\right) \not q S(p-k) .
$$

Substituindo a definição do propagador do bárion dada em (3.13) na Eq. (A.1), temos que

$$
I=-\frac{\left(\not p^{\prime}-\not k+m_{\Lambda}\right) \not q\left(\not p-\not k+m_{\Lambda}\right)}{\left[\left(p^{\prime}-k\right)^{2}-m_{\Lambda}^{2}\right]\left[(p-k)^{2}-m_{\Lambda}^{2}\right]}
$$

Para desenvolver esta expressão, aplicamos a distributiva no numerador, agrupamos os termos semelhantes,

$$
\begin{aligned}
\left(\not p^{\prime}-\not k+m_{\Lambda}\right) \not q\left(\not p-\not k+m_{\Lambda}\right)= & \left(\not p^{\prime} \not q \not p+m_{\Lambda}^{2} \not q\right)+m_{\Lambda}\left(\not p^{\prime} \not q+\not q \not p\right) \\
& -m_{\Lambda}(\not k \not q+\not q \not k)-\left(\not p^{\prime} \not q \not k+\not k \not q \not p\right) \\
& +(\not k \not q \not k)
\end{aligned}
$$

e, em seguida, calculamos os termos do numerador usando que $q=p^{\prime}-p$ e que, para quaisquer quadrivetores $a$ e $b$, vale a relação

$$
\not a \not b+\not b \not a=2 a \cdot b .
$$


Com isso,

$$
\begin{aligned}
& \not p^{\prime} \not q \not p+m_{\Lambda}^{2} \not q=\not q\left(m_{\Lambda}^{2}-m_{N}^{2}\right) \\
& \left(\not p^{\prime} \not q+\not q \not p\right)=0 \\
& \not k \not q+\not q \not k=2 k \cdot q \\
& \not p^{\prime} \not q \not k+\not k \not q \not p=\left(2 k \cdot p^{\prime}\right) \not p-(2 k \cdot p) \not p^{\prime} \\
& \not k \not q \not k=2 q \cdot k \not k-k^{2} \not q .
\end{aligned}
$$

Substituindo estes resultados no numerador da Eq. (A.1) podemos finalmente escrever

$$
I=-\frac{\not q\left(m_{\Lambda}^{2}-m_{N}^{2}-k^{2}\right)-2 k \cdot p^{\prime}\left(\not p-\not k+m_{\Lambda}\right)+2 k \cdot p\left(\not p^{\prime}-\not k+m_{\Lambda}\right)}{\left[\left(p^{\prime}-k\right)^{2}-m_{\Lambda}^{2}\right]\left[(p-k)^{2}-m_{\Lambda}^{2}\right]}
$$

Vamos agora desenvolver o segundo termo da identidade (3.18) que queremos provar. Seja

$$
I I=i\left(S(p-k)-S\left(p^{\prime}-k\right)\right) .
$$

Substituindo a definição do propagador do bárion e tirando o mínimo nas duas frações, temos que

$$
I I=\frac{\left(\not p^{\prime}-\not k+m_{\Lambda}\right)\left[(p-k)^{2}-m_{\Lambda}^{2}\right]-\left(\not p-\not k+m_{\Lambda}\right)\left[\left(p^{\prime}-k\right)^{2}-m_{\Lambda}^{2}\right]}{\left[\left(p^{\prime}-k\right)^{2}-m_{\Lambda}^{2}\right]\left[(p-k)^{2}-m_{\Lambda}^{2}\right]} .
$$

Assim como no cálculo de $I$, vamos abrir o numerador e agrupar os termos semelhantes. Neste caso, precisamos usar somente que $q=p^{\prime}-p$. Com isso obtemos diretamente que

$$
I I=-\frac{\not q\left(m_{\Lambda}^{2}-m_{N}^{2}-k^{2}\right)-2 k \cdot p^{\prime}\left(\not p-\not k+m_{\Lambda}\right)+2 k \cdot p\left(\not p^{\prime}-\not k+m_{\Lambda}\right)}{\left[\left(p^{\prime}-k\right)^{2}-m_{\Lambda}^{2}\right]\left[(p-k)^{2}-m_{\Lambda}^{2}\right]}
$$

e, portanto, $I=I I$. Ou seja,

$$
S\left(p^{\prime}-k\right) \not q S(p-k)=i\left(S(p-k)-S\left(p^{\prime}-k\right)\right),
$$

como queríamos demostrar. 


\section{Apêndice B}

\section{Relações úteis}

- Parametrização de Schwinger [44]:

$$
\frac{1}{A^{n}}=\frac{1}{(n-1) !} \int_{0}^{\infty} d \alpha \alpha^{n-1} e^{-\alpha A}
$$

- Identidade de Gordon [45]:

$$
\bar{U}\left(p^{\prime}\right) \gamma_{\mu} U(p)=\frac{1}{2 m_{N}} \bar{U}\left(p^{\prime}\right)\left[\left(p+p^{\prime}\right)_{\mu}+i \sigma_{\mu \nu} q^{\nu}\right] U(p)
$$

- Integrais quadridimensionais [44]:

$$
\begin{gathered}
\int \frac{d^{4} k}{(2 \pi)^{4}} \exp \left(A k^{2}+2 B \cdot k\right)=\frac{i}{(4 \pi)^{2}} \frac{1}{A^{2}} \exp \left(\frac{-B^{2}}{A}\right) \\
\int \frac{d^{4} k}{(2 \pi)^{4}} k_{\mu} \exp \left(A k^{2}+2 B \cdot k\right)=-\frac{i}{(4 \pi)^{2}} \frac{B_{\mu}}{A^{3}} \exp \left(\frac{-B^{2}}{A}\right) \\
\int \frac{d^{4} k}{(2 \pi)^{4}} k_{\mu} k_{\nu} \exp \left(A k^{2}+2 B \cdot k\right)=\frac{i}{(4 \pi)^{2}}\left[\frac{B_{\mu} B_{\nu}}{A^{4}}-\frac{g_{\mu \nu}}{2 A^{3}}\right] \exp \left(\frac{-B^{2}}{A}\right)
\end{gathered}
$$




\section{Referências Bibliográficas}

[1] M. Gell-Mann, Phys. Lett 8, 214 (1964).

[2] G. Zweig, CERN Reports 8182/TH.401, 8419/TH.412 (1964).

[3] M. Breidenbach et al., Phys. Rev. Lett. 23, 935 (1969).

[4] T. P. Cheng, Phys. Rev. D13, 2161 (1976).

[5] H. Ohki et al., hep-lat/0806.4744v1.

[6] J. Ashman et al., EMC Collaboration, Phys. Lett. B206, 364 (1988).

[7] B. W. Filippone, X.-D. Ji, Adv. Nucl. Phys. 26, 1 (2001).

[8] D. B. Kaplan, A. Manohar, Nucl. Phys. B310, 527 (1988).

[9] R. D. McKeown, Phys. Lett. B219, 140 (1989).

[10] D. H. Beck, Phys. Lett. D39, 3248 (1989).

[11] B. Mueller et al., SAMPLE Collaboration, Phys. Rev. Lett. 78, 3824 (1997).

[12] D. T. Spayde et al., SAMPLE Collaboration, Phys. Rev. Lett. 84, 1106 (2000).

[13] D. T. Spayde et al., SAMPLE Collaboration, Phys. Lett. B583, 79 (2004).

[14] K. A. Aniol et al., HAPPEX Collaboration, Phys. Rev. Lett. 82, 1096 (1999).

[15] K. A. Aniol et al., HAPPEX Collaboration, Phys. Rev. C69, 065501 (2004).

[16] K. A. Aniol et al., HAPPEX Collaboration, nucl-ex/0506011v2.

[17] A. Acha et al., HAPPEX Collaboration, Phys. Rev. Lett. 98, 032301 (2007).

[18] F. E. Maas et al., A4 Collaboration, Phys. Rev. Lett. 93, 022002 (2004).

[19] F. E. Maas et al., A4 Collaboration, Phys. Rev. Lett. 94, 152001 (2005). 
[20] D. S. Armstrong et al., G0 Collaboration, nucl-ex/0506011.

[21] F. Carvalho, F.S. Navarra and M. Nielsen, Phys. Rev. C72, 068202 (2005).

[22] C. Göbel, (for the E791 Collaboration), hep-ex/0110052, AIP Conf. Proc. 619, 63 (2002).

[23] S. F. Pate, D. W. McKee, V. Papavassiliou, hep-ex/0805.2889v1.

[24] L. A. Ahrens et al., E734 Collaboration, Phys. Rev. D35, 785 (1987).

[25] M. J. Musolf et al., Phys. Rep. 239, 1 (1994).

[26] F. Halzen, A. D. Martin, Quarks and Leptons, John Willey \& Sons, New York (1984).

[27] R. G. Sachs, Phys. Rev. 126, 2256 (1962).

[28] F. J. Ernst, R. G. Sachs, K. C. Wali, Phys. Rev. 119, 1105 (1960).

[29] W. M. Yao et al., J. Phys. G33 1 (2006).

[30] C. F. Perdrisat, V. Punjabi, M. Vanderhaeghen, Prog. Part. Nucl. Phys. 59, 694 (2007).

[31] T. M. Ito et al., SAMPLE Collaboration, Phys. Rev. Lett. 92, 102003 (2004).

[32] K. A. Aniol et al., HAPPEX Collaboration, nucl-ex/0506010v1.

[33] W. Meltnichouk and M. Malheiro, Phys. Rev. C55, 431 (1997).

[34] Y. Oh, H. Kim, hep-ph/0605105v1.

[35] B. Holzencamp, K. Holinde, J. Speth, Nucl. Phys. A500, 485 (1989).

[36] M. E. Peskin. D. V. Schroeder, An Introduction to Quantum Field Theory, AddisonWesley Pub. Co., New York, (1995).

[37] K. Ohta, Phys. Rev. C40, 1335 (1989).

[38] F. Carvalho, D. M. T. Leite, F. S. Navarra, M. Nielsen, Int. J. Mod. Phys. E16, 2880 (2007).

[39] V.G.J. Stoks, Th.A. Rijken, Phys. Rev. C59, 3009 (1999).

[40] J. Haidenbauer, U. G. Meissner, Phys. Rev. C72, 044005 (2005). 
[41] J.J. Kelly, Phys. Rev. C70, 068202 (2004).

[42] D. B. Leinweber et al., Phys. Rev. Lett. 94, 212001 (2005).

[43] D. H. Beck, R. D. McKeown, Annu. Rev. Nucl. Part. Sci. 51, 189 (2001).

[44] J. C. Collins, Renormalization, Cambridge University Press, New York (1984).

[45] C. Itzykson, J. Zuber, Quantum Field Theory, McGraw-Hill, Singapore (1980). 